\title{
A Bayesian Level Set Method for Geometric Inverse Problems
}

\author{
Marco A. Iglesias \\ School of Mathematical Sciences, University of Nottingham, Nottingham NG7 2RD, UK \\ marco.iglesias@ nottingham.ac.uk \\ Yulong $\mathrm{Lu}^{\dagger}$ \\ Mathematics Institute, University of Warwick, Coventry CV4 7AL, UK \\ † corresponding author. yulong.lu@warwick.ac.uk \\ A. M. Stuart \\ Mathematics Institute, University of Warwick, Coventry CV4 7AL, UK \\ a.m.stuart@warwick.ac.uk
}

\begin{abstract}
We introduce a level set based approach to Bayesian geometric inverse problems. In these problems the interface between different domains is the key unknown, and is realized as the level set of a function. This function itself becomes the object of the inference. Whilst the level set methodology has been widely used for the solution of geometric inverse problems, the Bayesian formulation that we develop here contains two significant advances: firstly it leads to a well-posed inverse problem in which the posterior distribution is Lipschitz with respect to the observed data, and may be used to not only estimate interface locations, but quantify uncertainty in them; and secondly it leads to computationally expedient algorithms in which the level set itself is updated implicitly via the MCMC methodology applied to the level set function - no explicit velocity field is required for the level set interface. Applications are numerous and include medical imaging, modelling of subsurface formations and the inverse source problem; our theory is illustrated with computational results involving the last two applications.
\end{abstract}

\section{Introduction}

Geometric inverse problems, in which the interfaces between different domains are the primary unknown quantities, are ubiquitous in applications including medical imaging problems such as EIT [10] and subsurface flow [6]; they also have an intrinsically interesting mathematical structure [35]. In many such applications the data is sparse, so that the problem is severely under-determined, and noisy. For both these reasons the Bayesian approach to the inverse problem is attractive as the probabilistic formulation allows for regularization of the under-determined, and often ill-posed, inversion via the introduction of priors, and simultaneously deals with the presence of noise in the 
observations [37, 49]. The level set method has been a highly successful methodology for the solution of classical, non-statistical, inverse problems for interfaces since the seminal paper of Santosa [47]; see for example [18, 14, 15, 51, 38, 39, 50, 16, 23, 24, 52, 34, 3, 7] and for related Bayesian level set approaches see [53, 42, 41, 45]. For interface problems, the phase field approach[22, 16] is sometimes used as an alternative to the level set method; in this approach the recovered interfaces between different phases are smeared out. Also, recently the Bayesian framework of [49] is adopted for the solution of inverse shape reconstruction in acoustic scattering problems [13].

In this paper we marry the level set approach with the Bayesian approach to geometric inverse problems. This leads to two significant advances: firstly it leads to a well-posed inverse problem in which the posterior distribution is Lipschitz with respect to the observed data, in the Hellinger metric - there is no analogous well-posedness theory for classical level set inversion; and secondly it leads to computationally expedient algorithms in which the interfaces are updated implicitly via the Markov chain Monte Carlo (MCMC) methodology applied to the level set function - no explicit velocity field is required for the level set interface. We highlight that the recent paper [52] demonstrates the potential for working with regularized data misfit minimization in terms of a level set function, but is non-Bayesian in its treatment of the problem, using instead simulated annealing within an optimization framework. On the other hand the paper [53] adopts a Bayesian approach and employs the level set method, but requires a velocity field for propagation of the interface and does not have the conceptual and implementational simplicity, as well as the underlying theoretical basis, of the method introduced here. The papers [42, 41, 45], whilst motivated by the Bayesian approach, use the ensemble Kalman filter and are therefore not strictly Bayesian - the method does not deliver a provably reliable approximation of the posterior distribution except for linear Gaussian inverse problems.

The key idea which underpins our work is this. Both the theory and computational practice of the level set method for geometric inverse problems is potentially hampered by the fact that the mapping from the space of the level set function to the physical parameter space is discontinuous. This discontinuity occurs when the level set function is flat at the critical levels, and in particular where the desired level set has non-zero Lebesgue measure. This is dealt with in various ad hoc ways in the applied literature. The beauty of the Bayesian approach is that, with the right choice of prior in level set space, these discontinuities have probability zero. As a result a well-posedness theory (the posterior is Lipschitz in the data) follows automatically, and computational algorithms such as MCMC may be formulated in level set space. We thus have practical algorithms which are simultaneously founded on a sound theoretical bedrock.

In section 2 we aim to build up a mathematical framework for Bayesian level set inversion. To do this, we first set up the inverse problem of interest in section 2.1 where the unknown is an interface. To describe the geometry of the interface, a level set map is introduced in section 2.2, whereby the inverse problem is reformulated in terms of a level set function which is thresholded to define the interfaces. Section 2.3 concerns the Bayesian approach to the inverse problem. Under certain 
assumptions on the negative log-likelihood function, it is shown that the posterior distribution exists and is stable with respect to perturbation of data; see the statements in Theorem 2.3. Since the well-posedness of the Bayesian inverse problem relies mostly on the almost sure continuity of the level set map, we discuss this issue thoroughly in section 2.4. To be specific, we provide a complete characterization for the discontinuity set of the level set map (in Proposition 2.6) and demonstrate the existence of Gaussian priors for which this discontinuity set is a probability zero event (in Proposition 2.8). In section 3 we describe two examples - inverse gravimetry and permeability determination in groundwater flow - which can be shown to satisfy the theoretical framework of the preceding section and hence for which there is a well-posed inverse problem for the level set function. Section 4 contains numerical experiments for both of these examples, demonstrating the potential of the methodology, and also highlighting important questions for future research. We conclude in section 5, and then the two appendices contain various technical details and proofs which have been deferred in order to maintain the flow of ideas in the main body of the article.

\section{Bayesian Level Set Inversion}

\subsection{The Inverse Problem}

This paper is concerned with inverse problems of the following type: recover function $\kappa \in X:=$ $L^{q}(D ; \mathbb{R}), D$ a bounded open set in $\mathbb{R}^{2}$, from a finite set of noisily observed linear functionals $\left\{\mathcal{O}_{j}\right\}_{j=1}^{J}$ of $p \in V$, for some Banach space $V$, where $p=G(\kappa)$ for nonlinear operator $G \in C(X, V)$. Typically, for us, $\kappa$ will represent input data for a partial differential equation (PDE), $p$ the solution of the PDE and $G$ the solution operator mapping the input $\kappa$ to the solution $p$. Collecting the linear functionals into a single operator $\mathcal{O}: V \rightarrow \mathbb{R}^{J}$ and assuming additive noise $\eta \in \mathbb{R}^{J}$ we obtain the inverse problem of finding $\kappa$ from $y$ where

$$
y=(\mathcal{O} \circ G)(\kappa)+\eta .
$$

Since the composite mapping $\mathcal{O} \circ G$ is continuous from $X$ to $\mathbb{R}^{J}$, identifying $\kappa$ in the above underdetermined inverse problem is well-adapted to both the classical [25] and Bayesian [21] approaches to regularized inversion. However interest is in geometric inverse problems using the level set formulation. For such problems, the mapping from the level set function to the data is discontinuous. Classical regularization methods have problems in this situation; Example 2.1 below is an example of such a difficulty. However, we will demonstrate that formulating the inverse problem from the Bayesian point of view alleviates these issues and leads to a well-posed inverse problem. 


\subsection{Level Set Parameterization}

Assume that the physical parameter $\kappa$ of the inverse problem is known a priori to have the form

$$
\kappa(x)=\sum_{i=1}^{n} \kappa_{i} \mathbb{I}_{D_{i}}(x) ;
$$

here $\mathbb{I}_{D}$ denotes the indicator function of subset $D \subset \mathbb{R}^{2},\left\{D_{i}\right\}_{i=1}^{n}$ are subsets of $D$ such that $\cup_{i=1}^{n} \overline{D_{i}}=\bar{D}$ and $D_{i} \cap D_{j}=\varnothing$, and the $\left\{\kappa_{i}\right\}_{i=1}^{n}$ are known positive constants. Generalization to the $\kappa_{i}$ being unknown constants, or unknown smooth functions on each domain $D_{i}$, are possible but will not be considered explicitly in this paper. Our focus is on the geometry of the interfaces implied by the $D_{i}$. In this setting the $D_{i}$ become the primary unknowns and the level set method is natural. Given integer $n$ fix the constants $c_{i} \in \mathbb{R}$ for $i=0, \cdots, n$ with $-\infty=c_{0}<c_{1}<\cdots<c_{n}=\infty$ and consider a real-valued continuous level set function $u: D \rightarrow \mathbb{R}$. We can then define the $D_{i}$ by

$$
D_{i}=\left\{x \in D \mid c_{i-1} \leq u(x)<c_{i}\right\}
$$

It follows that $D_{i} \cap D_{j}=\varnothing$ for $i, j \geq 1, i \neq j$. For later use define the $i$-th level set $D_{i}^{0}=$ $\overline{D_{i}} \cap \overline{D_{i+1}}=\left\{x \in D \mid u(x)=c_{i}\right\}$. Let $U=C(\bar{D} ; \mathbb{R})$ and, given the positive constants $\left\{\kappa_{i}\right\}_{i=1}^{n}$, we define the level set map $F: U \rightarrow X$ by

$$
(F u)(x) \rightarrow \kappa(x)=\sum_{i=1}^{n} \kappa_{i} \mathbb{I}_{D_{i}}(x) .
$$

We may now define $\mathcal{G}=\mathcal{O} \circ G \circ F: U \rightarrow \mathbb{R}^{J}$ and reformulate the inverse problem in terms of the level set function $u$ : find $u$ from $y$ where

$$
y=\mathcal{G}(u)+\eta
$$

However, because $F: U \rightarrow X$, and hence $\mathcal{G}: U \rightarrow \mathbb{R}^{J}$, is discontinuous, the classical regularization theory for this form of inverse problem is problematic; this can be seen from the following example.

Example 2.1. Consider the inverse problem (2.5) where the level set map is given by the binary cut-off, i.e.

$$
(F u)(x)=\mathbb{I}_{u \geq 0}(x) .
$$

Classical regularization methods seek the solution to the following minimization problem:

$$
\inf _{u \in E} I(u):=\inf _{u \in E}|y-\mathcal{G}(u)|^{2}+\|u\|_{E}^{p}
$$

where $E$ is some Banach space and $p \geq 1$. For instance, $E$ could be a Sobolev space and $p=$ 2 (Tikhonov-Phillips regularization) or the space of functions of bounded variations and $p=1$ (total variation regularization). In the case of a Gaussian prior and an appropriate Sobolev norm, 
this variational problem will correspond to a maximum a posteriori (MAP) estimator [37] and the analysis to follow shows a drawback of the MAP estimator in the context of level set thresholding.

Due to the discontinuity of $F$, we now show that the only possible minimizer of (2.7) is zero. In fact, suppose that $0 \neq \bar{u} \in E$ is a minimizer of $I$. We define $u_{\varepsilon}=\varepsilon \bar{u}$. Clearly $u_{\varepsilon}$ and $\bar{u}$ have the same zero level set when $\varepsilon>0$. Then if $0<\varepsilon<1$,

$$
|y-\mathcal{G}(\bar{u})|^{2}=\left|y-\mathcal{G}\left(u_{\varepsilon}\right)\right|^{2} \quad \text { and } \quad\left\|u_{\varepsilon}\right\|_{E}=\varepsilon\|\bar{u}\|_{E},
$$

which implies that $I\left(u_{\varepsilon}\right)<I(\bar{u})$ and hence contradicts with the assumption that $\bar{u}$ is a minimizer. From (2.6), we see that the upper level set of the zero function is the whole domain, which does not provide any information about the geometry.

Whilst the current state of the art for Bayesian regularization assumes continuity of $\mathcal{G}$ for inverse problems of the form (2.5), we will demonstrate that the Bayesian setting can be generalized to level set inversion. This will be achieved by a careful understanding of the discontinuity set for $F$, and an understanding of probability measures for which this set is a measure zero event.

\subsection{Well-Posed Bayesian Inverse Problem}

We now formulate the Bayesian approach to finding $u$ from $y$ given by (2.5). All quantities are treated as random variables and we seek to find the posterior probability distribution on $u$ given $y$, given a prior probability distribition on $u$ and an independent probabilistic specification of the noise $\eta$. Let $U$ denote a separable Banach space and define a complete probability space $\left(U, \Sigma, \mu_{0}\right)$ for the unknown $u$. Here $\Sigma$ and $\mu_{0}$ are the sigma algebra and prior probability measure, respectively. (In our applications $U$ will be the space $C(\bar{D} ; \mathbb{R})$ but we state our main theorem in more generality). Assume that the noise $\eta$ is a random draw from the centered Gaussian $\mathbb{Q}_{0}:=\mathcal{N}(0, \Gamma)$. Allowing for non-Gaussian $\eta$ is also possible, as is dependence between $\eta$ and $u$; however we elect to keep the presentation simple. We may now define the joint random variable $(u, y) \in U \times \mathbb{R}^{J}$. The posterior probability distribution $\mu^{y}$ on the random variable $u \mid y$ describes our probabilistic knowledge about $u$ on the basis of the measurements $y$ given by (2.5) and the prior information $\mu_{0}$ on $u$. In the case where the map $\mathcal{G}$ is continuous, one can apply an infinite dimensional version of Bayes theorem [49] to show that the posterior $\mu^{y}$ exists and has the density with respect to the prior of the form

$$
\frac{d \mu^{y}}{d \mu_{0}}(u)=\frac{1}{Z} \exp (-\Phi(u ; y)),
$$

where $Z$ is the normalization constant. To extend the theory to allowing discontinuous $\mathcal{G}$, we now state a set of assumptions for the potential $\Phi$, under which the posterior distribution is well-defined via its density with respect to the prior distribution, and is Lipschitz in the Hellinger metric, with respect to data $y$. These assumptions will be verified for the level set formulation of interest to us.

Assumptions 2.2. The function $\Phi: U \times \mathbb{R}^{J} \rightarrow \mathbb{R}$ and probability measure $\mu_{0}$ on the measure space $(U, \Sigma)$ satisfy the following properties: 
1. for every $r>0$ there is a $K=K(r)$ such that, for all $u \in U$ and all $y \in \mathbb{R}^{J}$ with $|y|_{\Gamma}<r$,

$$
0 \leq \Phi(u ; y) \leq K
$$

2. for any fixed $y \in \mathbb{R}^{J}, \Phi(\cdot ; y): U \rightarrow \mathbb{R}$, is continuous $\mu_{0}$-almost surely on the complete probability space $\left(U, \Sigma, \mu_{0}\right)$;

3. for $y_{1}, y_{2} \in \mathbb{R}^{J}$ with $\max \left\{\left|y_{1}\right|_{\Gamma},\left|y_{2}\right|_{\Gamma}\right\}<r$, there exists a $C=C(r)$ such that, for all $u \in U$,

$$
\left|\Phi\left(u ; y_{1}\right)-\Phi\left(u ; y_{2}\right)\right| \leq C\left|y_{1}-y_{2}\right|_{\Gamma}
$$

For our Bayesian level set inverse problem with finite observations and noise $\eta \sim \mathbb{Q}_{0}$, the function $\Phi: U \times \mathbb{R}^{J} \rightarrow \mathbb{R}^{+}$has the least squares form

$$
\Phi(u ; y)=\frac{1}{2}|y-\mathcal{G}(u)|_{\Gamma}^{2}
$$

with $|\cdot|_{\Gamma}:=\left|\Gamma^{-\frac{1}{2}} \cdot\right|$ and $\mathcal{G}=\mathcal{O} \circ G \circ F$. Clearly $\Phi$ defined in (2.9) satisfies the first and the last item of Assumption 2.2. We will show in the next section that for some model problems, the second item of Assumption 2.2 will also be fulfilled by $\Phi$ in (2.9).

Recall that the Hellinger distance between $\mu$ and $\mu^{\prime}$ is defined as

$$
d_{\mathrm{Hell}}\left(\mu, \mu^{\prime}\right)=\left(\frac{1}{2} \int_{U}\left(\sqrt{\frac{\mathrm{d} \mu}{\mathrm{d} \nu}}-\sqrt{\frac{\mathrm{d} \mu^{\prime}}{\mathrm{d} \nu}}\right)^{2} \mathrm{~d} \nu\right)^{\frac{1}{2}}
$$

for any measure $\nu$ with respect to which $\mu$ and $\mu^{\prime}$ are absolutely continuous; the Hellinger distance is, however, independent of which reference measure $\nu$ is chosen. We have the following:

Theorem 2.3. Assume that the least squares function $\Phi: U \times \mathbb{R}^{J} \rightarrow \mathbb{R}$ given by (2.9) and the probability measure $\mu_{0}$ on the measure space $(U, \Sigma)$ satisfy Assumptions 2.2. Then $\mu^{y} \ll \mu_{0}$ with Radon-Nikodym derivative

$$
\frac{\mathrm{d} \mu^{y}}{\mathrm{~d} \mu_{0}}=\frac{1}{Z} \exp (-\Phi(u ; y))
$$

where, for $y$ almost surely,

$$
Z:=\int_{U} \exp (-\Phi(u ; y)) \mu_{0}(d u)>0 .
$$

Furthermore $\mu^{y}$ is locally Lipschitz with respect to $y$, in the Hellinger distance: for all $y, y^{\prime}$ with $\max \left\{|y|_{\Gamma},\left|y^{\prime}\right|_{\Gamma}\right\}<r$, there exists a $C=C(r)>0$ such that

$$
d_{\mathrm{Hell}}\left(\mu^{y}, \mu^{y^{\prime}}\right) \leq C\left|y-y^{\prime}\right|_{\Gamma}
$$

This implies that, for all $f \in L_{\mu_{0}}^{2}(U ; S)$ for separable Banach space $S$,

$$
\left\|\mathbb{E}^{\mu^{y}} f(u)-\mathbb{E}^{\mu^{y^{\prime}}} f(u)\right\|_{S} \leq C\left|y-y^{\prime}\right|
$$


Remarks 2.4. - The interpretation of this result is very natural, linking the Bayesian picture with least squares minimization: the posterior measure is large on sets where the least squares function is small, and vice-versa, all measured relative to the prior $\mu_{0}$.

- The key technical advance in this theorem over existing theories overviewed in [21] is that $\Phi(\cdot ; y)$ is only continuous $\mu_{0}$-almost surely; existing theories typically use that $\Phi(\cdot ; y)$ is continuous everywhere on $U$ and that $\mu_{0}(U)=1$; these existing theories cannot be used in the level set inverse problem, because of discontinuities in the level set map. Once the technical Lemma 6.1 has been established, which uses $\mu_{0}$-almost sure continuity to establish measurability, the proof of the theorem is a straightforward application of existing theory; we therefore defer it to Appendix 1.

- Stability estimates about the distance of level sets can be obtained by choosing $f$ carefully in (2.11). Indeed, consider $f: U \mapsto L^{1}(D)$ given by

$$
f(u)(x):=\mathbb{I}_{D_{i}}(x)
$$

where $D_{i}$ is defined in terms of $u$ as in (2.3). Obviously $f \in L_{\mu_{0}}^{2}\left(U ; L^{1}(D)\right)$ since the indicator function is uniformly bounded. Then one can read from (2.11) that the $L^{1}$-norm of mean indicator function of the set $D_{i}$ under the posterior measure is Lipschitz continuous with respect to the data. Note that this does not give exactly the symmetric difference of the two mean level sets since indicator functions are averaged first. However, it does reflect stability of geometric reconstructions in an averaged sense.

- What needs to be done to apply this theorem in our level set context is to identify the sets of discontinuity for the map $\mathcal{G}$, and hence $\Phi(\cdot ; y)$, and then construct prior measures $\mu_{0}$ for which these sets have measure zero. We study these questions in general terms in the next two subsections, and then, in the next section, demonstrate two test model PDE inverse problems where the general theory applies.

- The consequences of this result are wide-ranging, and we name the two primary ones: firstly we may apply the mesh-independent MCMC methods overviewed in [19] to sample the posterior distribution efficiently; and secondly the well-posedness gives desirable robustness which may be used to estimate the effect of other perturbations, such as approximating $G$ by a numerical method, on the posterior distribution [21].

\subsection{Discontinuity Sets of $F$}

We return to the specific setting of the level set inverse problem where $U=C(\bar{D} ; \mathbb{R})$. Consider the level set map $F: U \rightarrow L^{q}(D ; \mathbb{R})$ with $1 \leq q \leq \infty$. First we note that it is not suitable to discuss the continuity of $F$ by choosing $L^{\infty}(D, \mathbb{R})$ as the range space, simply because $F$ could be 
discontinuous at very nice functions. We illustrate this point by means of the following elementary example.

Example 2.5. Let $U=C([-1,1] ; \mathbb{R})$ and define the level set map $F: U \mapsto L^{\infty}(-1,1)$ by setting $F(u)(x)=\mathbb{I}_{\{u \geq 0\}}(x)$. Consider the linear function $u(x)=x$ and a sequence $u_{n}(x)=u(x)+1 / n$ for $n \in \mathbb{N}$. Clearly $u_{n} \rightarrow u$ in $C([-1,1] ; \mathbb{R})$. However, it is easy to see that $\left\|F\left(u_{n}\right)-F(u)\right\|_{L^{\infty}(-1,1)}=$ $1 \nrightarrow 0$.

However as a mapping $F: U \rightarrow L^{q}(D ; \mathbb{R})$ for $q<\infty$ the situation is much better. Denoting by $m(A)$ the Lebesgue measure of a set $A \subset \mathbb{R}^{2}$, we have the following.

Proposition 2.6. For $u \in C(\bar{D})$ and $1 \leq q<\infty$, the level set map $F: C(\bar{D}) \rightarrow L^{q}(D)$ is continuous at $u$ if and only if $m\left(D_{i}^{0}\right)=0$ for all $i=1, \cdots, n-1$.

Remark 2.7. The fact that the continuity of level set map is related to the Lebesgue measure of the corresponding level sets has been observed already, see e.g. [27, Section 2.2]. However, we are not aware of any formal proof in the literature. Therefore we provide the complete proof below.

Proof of Proposition 2.6. “ $\Longleftarrow$." Let $\left\{u_{\varepsilon}\right\}$ denote any approximating family of level set functions with limit $u$ as $\varepsilon \rightarrow 0$ in $C(\bar{D} ; \mathbb{R}):\left\|u_{\varepsilon}-u\right\|_{C(\bar{D})}<\varepsilon \rightarrow 0$. Let $D_{i, \varepsilon}, D_{i, \varepsilon}^{0}$ be the sets defined in (2.3) associated with the approximating level set function $u_{\varepsilon}$ and define $\kappa=F(u)$ by (2.4) and, similarly, $\kappa_{\varepsilon}:=F\left(u_{\varepsilon}\right)$. Let $m(A)$ denote the Lebesgue measure of the set $A$.

Suppose that $m\left(D_{i}^{0}\right)=0, i=1, \cdots, n-1$. Let $\left\{u_{\varepsilon}\right\}$ be the above approximating functions. We shall prove $\left\|\kappa_{\varepsilon}-\kappa\right\|_{L^{q}(D)} \rightarrow 0$. In fact, we can write

$$
\begin{aligned}
\kappa_{\varepsilon}(x)-\kappa(x) & =\sum_{i=1}^{n} \sum_{j=1}^{n}\left(\kappa_{i}-\kappa_{j}\right) \mathbb{I}_{D_{i, \varepsilon} \cap D_{j}}(x) \\
& =\sum_{i, j=1, i \neq j}^{n}\left(\kappa_{i}-\kappa_{j}\right) \mathbb{I}_{D_{i, \varepsilon} \cap D_{j}}(x) .
\end{aligned}
$$

By the definition of $u_{\varepsilon}$, for any $x \in D$

$$
u(x)-\varepsilon<u_{\varepsilon}(x)<u(x)+\varepsilon
$$

Thus for $|j-i|>1$ and $\varepsilon$ sufficiently small, $D_{i, \varepsilon} \cap D_{j}=\varnothing$. For the case that $|i-j|=1$, from (2.13), it is easy to verify that

$$
\begin{aligned}
D_{i, \varepsilon} \cap D_{i+1} \subset \widetilde{D}_{i, \varepsilon} & :=\left\{x \in D \mid c_{i} \leq u(x)<c_{i}+\varepsilon\right\} \rightarrow D_{i}^{0}, i=1, \cdots, n-1 \\
D_{i, \varepsilon} \cap D_{i-1} \subset \widehat{D}_{i-1, \varepsilon} & :=\left\{x \in D \mid c_{i-1}-\varepsilon<u(x)<c_{i-1}\right\} \rightarrow \varnothing, i=2, \cdots n
\end{aligned}
$$

as $\varepsilon \rightarrow 0$. By this and the assumption that $m\left(D_{i}^{0}\right)=0$, we have that $m\left(D_{i, \varepsilon} \cap D_{j}\right) \rightarrow 0$ if $i \neq j$. Furthermore, the Lebesgue's dominated convergence theorem yields

$$
\left\|\kappa_{\varepsilon}-\kappa\right\|_{L^{q}(D)}^{q}=\sum_{i, j=1, i \neq j}^{n} \int_{D_{i, \varepsilon} \cap D_{j}}\left|\kappa_{i}-\kappa_{j}\right|^{q} \mathrm{~d} x \rightarrow 0
$$


as $\varepsilon \rightarrow 0$. Therefore, $F$ is continuous at $u$.

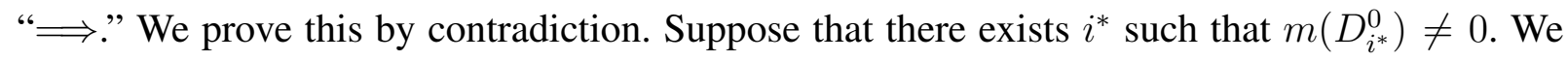
define $u_{\varepsilon}:=u-\varepsilon$, then it is clear that $\left\|u_{\varepsilon}-u\right\|_{C(\bar{D})} \rightarrow 0$ as $\varepsilon \rightarrow 0$. By the same argument used in proving the sufficiency,

$\left\|\kappa_{\varepsilon}-\kappa\right\|_{L^{q}(D)}^{q}=\sum_{i=1}^{n-1} \int_{\widetilde{D}_{i, \varepsilon} \cup \widehat{D}_{i, \varepsilon}}\left|\kappa_{i+1}-\kappa_{i}\right|^{q} \mathrm{~d} x \rightarrow \sum_{i=1}^{n-1} \int_{D_{i}^{0}}\left|\kappa_{i+1}-\kappa_{i}\right|^{q} \mathrm{~d} x>\int_{D_{i^{*}}^{0}}\left|\kappa_{i^{*}+1}-\kappa_{i^{*}}\right|^{q} \mathrm{~d} x>0$

where we have used $m\left(D_{i^{*}}^{0}\right) \neq 0$ in the last inequality. However, this contradicts with the assumption that $F$ is continuous at $u$.

For the inverse gravimetry problem considered in the next section the space $X$ is naturally $L^{2}(D ; \mathbb{R})$ and we will be able to directly use the preceding proposition to establish almost sure continuity of $F$ and hence $\mathcal{G}$. For the groundwater flow inverse problem the space $X$ is naturally $L^{\infty}(D ; \mathbb{R})$ and we will not be able to use the proposition in this space to establish almost sure continuity of $F$. However, we employ recent Lipschitz continuity results [9] for $G$ on $L^{q}(D ; \mathbb{R}), q<\infty$ to establish the almost sure continuity of $\mathcal{G}$.

\subsection{Prior Gaussian Measures}

Let $D$ denote a bounded open subset of $\mathbb{R}^{2}$. For our applications we will use the following two constructions of Gaussian prior measures $\mu_{0}$ which are Gaussian $\mathcal{N}\left(0, \mathcal{C}_{i}\right), i=1,2$ on Hilbert function space $\mathcal{H}_{i}, i=1,2$.

- $\mathcal{N}\left(0, \mathcal{C}_{1}\right)$ on

$$
\left.\mathcal{H}_{1}:=\left\{u: D \rightarrow \mathbb{R} \mid u \in L^{2}(D ; \mathbb{R})\right), \int_{D} u(x) \mathrm{d} x=0\right\}
$$

where

$$
\mathcal{C}_{1}=\mathcal{A}^{-\alpha} \quad \text { with } \alpha>1 \text { and } \quad \mathcal{A}:=-\Delta
$$

with domain

$$
D(\mathcal{A}):=\left\{u: D \rightarrow \mathbb{R} \mid u \in H^{2}(D ; \mathbb{R}), \nabla u \cdot \nu=0 \text { on } \partial D \text { and } \int_{D} u(x) \mathrm{d} x=0\right\} .
$$

Here $\nu$ denotes the outward normal.

- $\mathcal{N}\left(0, \mathcal{C}_{2}\right)$ on $\mathcal{H}_{2}:=L^{2}(D ; \mathbb{R})$ with $\mathcal{C}_{2}: \mathcal{H}_{2} \rightarrow \mathcal{H}_{2}$ being the integral operator

$$
\mathcal{C}_{2} \phi(x)=\int_{D} c(x, y) \phi(y) \mathrm{d} y \text { with } c(x, y)=\exp \left(-\frac{|x-y|^{2}}{L^{2}}\right)
$$

In fact, in the inverse model arising from groundwater flow studied in [32, 31], the Gaussian measure $\mathcal{N}\left(0, \mathcal{C}_{1}\right)$ was taken as the prior measure for the logarithm of the permeability. On the other hand the 
Gaussian measure $\mathcal{N}\left(0, \mathcal{C}_{2}\right)$ is widely used to model the earth's subsurface [44] as draws from this measure generate smooth random functions in which the parameter $L$ sets the spatial correlation length. For both of these measures it is known that, under suitable conditions on the domain $D$, draws are almost surely in $C(\bar{D} ; \mathbb{R})$; see [21], Theorems 2.16 and 2.18 for details.

Since $\alpha>1$ in (2.16), the Gaussian random function with measure $\mu_{0}$ defined in either case above has the property that, for $U:=C(\bar{D} ; \mathbb{R}), \mu_{0}(U)=1$. Since $U$ is a separable Banach space $\mu_{0}$ can be redefined as a Gaussian measure on $U$. Furthermore it is possible to define the appropriate $\sigma$-algebra $\Sigma$ in such a way that $\left(U, \Sigma, \mu_{0}\right)$ is a complete probability space; for details see Appendix 2. We have the following, which is a subset of what is proved in Proposition 7.2.

Proposition 2.8. Consider a random function $u$ drawn from one of the Gaussian probability measures $\mu_{0}$ on $U$ given above. Then $m\left(D_{i}^{0}\right)=0, \mu_{0}$-almost surely, for $i=1, \cdots, n$.

This, combined with Proposition 2.6, is the key to making a rigorous well-posed formulation of Bayesian level set inversion. Together the results show that priors may be constructed for which the problematic discontinuities in the level set map are probability zero events. In the next section we demonstrate how the theory may be applied, by considering two examples.

\section{Examples}

\subsection{Test Model 1 (Inverse Potential Problem)}

Let $D \subset \mathbb{R}^{2}$ be a bounded open set with Lipschitz boundary. Consider the PDE

$$
\Delta p=\kappa \quad \text { in } D, \quad p=0 \quad \text { on } \partial D
$$

If $\kappa \in X:=L^{2}(D)$ it follows that there is a unique solution $p \in H_{0}^{1}(D)$. Furthermore $\Delta p \in L^{2}(D)$, so that the Neumann trace can be defined in $V:=H^{-\frac{1}{2}}(\partial D)$ by the following Green's formula:

$$
\left\langle\frac{\partial p}{\partial \nu}, \varphi\right\rangle_{\partial D}=\int_{D} \Delta p \varphi \mathrm{d} x+\int_{D} \nabla p \nabla \varphi \mathrm{d} x
$$

for $\varphi \in H^{1}(D)$. Here $\nu$ is the unit outward normal vector on $\partial D$ and $\langle\cdot, \cdot\rangle_{\partial D}$ denotes the dual pairing on the boundary. We can then define the bounded linear map $G: X \rightarrow V$ by $G(\kappa)=\frac{\partial p}{\partial \nu}$.

Now assume that the source term $\kappa$ has the form

$$
\kappa(x)=\mathbb{I}_{D_{1}}(x)
$$

for some $D_{1} \subseteq D$. The inverse potential problem is to reconstruct the support $D_{1}$ from measurements of the Neumann data of $p$ on $\partial D$. In the case where the Neumann data is measured everywhere on the boundary $\partial D$, and where the domain $D_{1}$ is assumed to be star-shaped with respect to its center of gravity, the inverse problem has a unique solution; see [35, 36] for details of this theory 
and see $[29,16]$ for discussion of numerical methods for this inverse problem. We will study the underdetermined case where a finite set of bounded linear functionals $\mathcal{O}_{j}: V \rightarrow \mathbb{R}$ are measured, noisily, on $\partial D$ :

$$
y_{j}=\mathcal{O}_{j}\left(\frac{\partial p}{\partial \nu}\right)+\eta_{j} .
$$

Concatenating we have $y=(\mathcal{O} \circ G)(\kappa)+\eta$. Representing the boundary of $D_{1}$ as the zero level set of a function $u \in U:=C(\bar{D} ; \mathbb{R})$ we write the inverse problem in the form (2.5):

$$
y=(\mathcal{O} \circ G \circ F)(u)+\eta .
$$

Since multiplicity and uncertainty of solutions are then natural, we will adopt a Bayesian approach.

Notice that the level set map $F: U \rightarrow X$ is bounded: for all $u \in U$ we have $\|F(u)\|_{X} \leq$ $\operatorname{Vol}(D):=\int_{D} d x$. Since $G: X \rightarrow V$ and $\mathcal{O}: V \rightarrow \mathbb{R}^{J}$ are bounded linear maps it follows that $\mathcal{G}=\mathcal{O} \circ G \circ F: U \rightarrow \mathbb{R}^{J}$ is bounded: we have constant $C^{+} \in \mathbb{R}^{+}$such that, for all $u \in U$, $|\mathcal{G}(u)| \leq C^{+}$. From this fact Assumptions 2.2(1) and (3) follow automatically. Since both $G: X \rightarrow$ $V$ and $\mathcal{O}: V \rightarrow \mathbb{R}^{J}$ are bounded, and hence continuous, linear maps, the discontinuity set of $\mathcal{G}$ is determined by the discontinuity set of $F: U \rightarrow X$. By Proposition 2.6 this is precisely the set of functions for which the measure of the level set $\{u(x)=0\}$ is zero. By Proposition 2.8 this occurs with probability zero for both of the Gaussian priors specified there and hence Assumptions 2.2(2) holds with these priors. Thus Theorem 2.3 applies and we have a well-posed Bayesian inverse problem for the level set function.

\subsection{Test Model 2 (Discontinuity Detection in Groundwater Flow)}

Consider the single-phase Darcy-flow model given by

$$
-\nabla \cdot(\kappa \nabla p)=f \quad \text { in } D, p=0 \quad \text { on } \partial D .
$$

Here $D$ is a bounded Lipschitz domain in $\mathbb{R}^{2}, \kappa$ the real-valued isotropic permeability function and $p$ the fluid pressure. The right hand side $f$ accounts for the source of groundwater recharge. Let $V=H_{0}^{1}(D ; \mathbb{R}), X=L^{\infty}(D ; \mathbb{R})$ and $V^{*}$ denote the dual space of $V$. If $f \in V^{*}$ and $X^{+}:=\{\kappa \in$ $\left.X: \operatorname{essinf}_{x \in D} \kappa(x) \geq \kappa_{\min }>0\right\}$ then $G: X^{+} \mapsto V$ defined by $G(\kappa)=p$ is Lipschitz continuous and

$$
\|G(\kappa)\|_{V}=\|p\|_{V} \leq\|f\|_{V^{*}} / \kappa_{\min } .
$$

We consider the practically useful situation in which the permeability function $\kappa$ is modelled as piecewise constant on different regions $\left\{D_{i}\right\}_{i=1}^{n}$ whose union comprise $D$; this is a natural way to characterize heterogeneous subsurface structure in a physically meaningful way. We thus have

$$
\kappa(x)=\sum_{i=1}^{n} \kappa_{i} \mathbb{I}_{D_{i}}(x)
$$


where $\left\{D_{i}\right\}_{i=1}^{n}$ are subsets of $D$ such that $\cup_{i=1}^{n} \overline{D_{i}}=\bar{D}$ and $D_{i} \cap D_{j}=\varnothing$, and where the $\left\{\kappa_{i}\right\}_{i=1}^{n}$ are positive constants. We let $\kappa_{\min }=\min _{i} \kappa_{i}$.

Unique reconstruction of the permeability in some situations is possible if the pressure $p$ is measured everywhere $[2,46]$. The inverse problem of interest to us is to locate the discontinuity set of the permeability from a finite set of measurements of the pressure $p$. Such problems have also been studied in the literature. For instance, the paper [50] considers the problem by using multiple level set methods in the framework of optimization; and in [33], the authors adopt a Bayesian approach to reconstruct the permeability function characterized by layered or channelized structures whose geometry can be parameterized finite dimensionally. As we consider a finite set of noisy measurements of the pressure $p$, in $V^{*}$, and the problem is underdetermined and uncertain, the Bayesian approach is again natural. We make the significant extension of [33] to consider arbitrary interfaces, requiring infinite dimensional parameterization: we introduce a level set parameterization of the domains $D_{i}$, as in (2.3) and (2.4).

Let $\mathcal{O}: V \rightarrow \mathbb{R}^{J}$ denote the collection of $J$ linear functionals on $V$ which are our measurements. Because of the estimate (3.5) it is straightforward to see that $\mathcal{G}=\mathcal{O} \circ G \circ F$ is bounded as a mapping from $U$ into $\mathbb{R}^{J}$ and hence that Assumptions 2.2(1) and (3) hold; it remains to establish (2). To that end, from now on we need slightly higher regularity on $f$. In particular, we assume that, for some $q>2, f \in W^{-1}\left(L^{q}(D)\right)$. Here the space $W^{-1}\left(L^{q}(D)\right):=\left(W_{0}^{1, q^{*}}(D)\right)^{*} \subset V^{*}$ for $q^{*}$ and $q$ conjugate: $1 / q+1 / q^{*}=1$. It is shown in [9] that there exits $q_{0}>2$ such that the solution of (3.4) satisfies

$$
\|\nabla p\|_{L^{q}(D)} \leq C\|f\|_{W^{-1}\left(L^{q}(D)\right)}
$$

for some $C<\infty$ provided $2 \leq q<q_{0}$. We assume that such a $q$ is chosen. It then follows that $G$ is Lipschitz continuous from $L^{r}$ to $V$ where $r:=2 q /(q-2) \in[2, \infty)$. To be precise, let $p_{i}$ be the solution to the problem (3.4) with $\kappa_{i}, i=1,2$. Then the following is proved in [9]: for any $q \geq 2$,

$$
\left\|p_{1}-p_{2}\right\|_{V} \leq \frac{1}{\kappa_{\min }}\left\|\nabla p_{1}\right\|_{L^{q}(D)}\left\|\kappa_{1}-\kappa_{2}\right\|_{L^{r}(D)}
$$

provided $\nabla p_{1} \in L^{q}(D)$.

Hence $G: L^{r}(D) \rightarrow V$ is Lipschitz under our assumption that $f \in W^{-1}\left(L^{q}(D)\right)$ for some $q \in(2, \infty)$. By viewing $F: U \rightarrow L^{r}(D)$, it follows from Proposition (2.6) and Proposition (2.8) that Assumptions (2.2) (2) holds with both Gaussian priors defined in subsection 2.5. As a consequence Theorem 2.3 also applies in the groundwater flow model.

\section{Numerical Experiments}

Application of the theory developed in subsection 2.3 ensures that, for the choices of Gaussian priors discussed in subsection 2.5, the posterior measure on the level set is well defined and thus suitable for numerical interrogation. In this section we display numerical experiments where we 
characterize the posterior measure by means of sampling with MCMC. In concrete we apply the preconditioned Crank-Nicolson (pCN) MCMC method explained in [19]. We start by defining this algorithm. Assume that we have a prior Gaussian measure $\mathcal{N}(0, \mathcal{C})$ on the level set function $u$ and a posterior measure $\mu^{y}$ given by (2.10). Define

$$
a(u, v)=\min \{1, \exp (\Phi(u)-\Phi(v))\}
$$

and generate $\left\{u^{(k)}\right\}_{k \geq 0}$ as follows:

Algorithm 4.1 (pCN-MCMC).

Set $k=0$ and pick $u^{(0)} \in X$.

1. Propose $v^{(k)}=\sqrt{\left(1-\beta^{2}\right)} u^{(k)}+\beta \xi^{(k)}, \quad \xi^{(k)} \sim \mathcal{N}(0, \mathcal{C})$.

2. Set $u^{(k+1)}=v^{(k)}$ with probability $a\left(u^{(k)}, v^{(k)}\right)$, independently of $\left(u^{(k)}, \xi^{(k)}\right)$.

3. Set $u^{(k+1)}=u^{(k)}$ otherwise.

4. $k \rightarrow k+1$ and return to 1 .

Then the resulting Markov chain is reversible with respect to $\mu^{y}$ and, provided it is ergodic, satisfies

$$
\frac{1}{K} \sum_{k=0}^{K} \varphi\left(u^{(k)}\right) \rightarrow \mathbb{E}^{\mu^{y}} \varphi(u)
$$

for any test function $\varphi$ with suitable regularity. Furthermore a central limit theorem determines the fluctuations around the limit, which are asymptotically of size $K^{-\frac{1}{2}}$.

\subsection{Aim of the experiments}

By means of the MCMC method described above we explore the Bayesian posterior of the level set function that we use to parameterize unknown geometry (or discontinuous model parameters) in the geometric inverse problems discussed in Section 3. The first experiment of this section concerns the inverse potential problem defined in subsection 3.1. The second and third experiments are concerned with the estimation of geologic facies for the groundwater flow model discussed in subsection 3.2. The main objective of these experiments is to display the capabilities of the level set Bayesian framework to provide an estimate, along with a measure of its uncertainty, of unknown discontinuous model parameters in these test models. We recall that for the inverse potential problem the aim is to estimate the support $D_{1}$ of the indicator function $\kappa(x)=\mathbb{I}_{D_{1}}(x)$, that defines the source term of the PDE (3.1), given data/observations from the solution of this PDE. Similarly, given data/observations from the solution of the Darcy flow model (3.4), we wish to estimate the interface between geologic facies $\left\{D_{i}\right\}_{i=1}^{n}$ corresponding to regions of different structural geology and which leads to a discontinuous permeability $\kappa(x)=\sum_{i=1}^{n} \kappa_{i} \mathbb{I}_{D_{i}}(x)$ in the flow model (3.4). 
In both test models, we introduce the level set function merely as an artifact to parameterize the unknown geometry (i.e. $D_{i}=\left\{x \in D \mid c_{i-1} \leq u(x)<c_{i}\right\}$ ), or equivalently, the resulting discontinuous field $\kappa(x)$. The Bayesian framework applied to this level-set parameterization then provides us with a posterior measure $\mu^{y}$ on the level set function $u$. The push-forward of $\mu^{y}$ under the level set map $F$ (2.4) results in a distribution on the discontinuous field of interest $\kappa$. This push-forward of the level set posterior $F^{*} \mu^{y}:=\mu^{y} \circ F^{-1}$ comprises the statistical solution of the inverse problem which may, in turn, be used for practical applications.

A secondary aim of the experiments is to explore the role of the choice of prior on the posterior. Because the prior is placed on the level set function, and not on the model paramerers of direct interest, this is a non-trivial question. To be concrete, the posterior depends on the Gaussian prior that we put on the level set. While the prior may incorporate our a priori knowledge concerning the regularity and the spatial correlation of the unknown geometry (or alternatively, the regions of discontinuities in the fields of interest) it is clear that such selection of the prior on the level set may have a strong effect on the resulting posterior $\mu^{y}$ and the corresponding push-forward $F^{*} \mu^{y}$. One of the key aspects of the subsequent numerical study is to understand the role of the selection of the prior on the level set functions in terms of the quality and efficiency of the solution to the Bayesian inverse problem as expressed via the push-forward of the posterior $F^{*} \mu^{y}$.

\subsection{Implementational aspects}

For the numerical examples of this section we consider synthetic experiments. The PDEs that define the forward models of subsection 3 (i.e. expressions (3.1) and (3.4)) are solved numerically, on the unit-square, with cell-centered finite differences [5]. In order to avoid inverse crimes [37], for the generation of synthetic data we use a much finer grid (size specified below) than the one of size $80 \times 80$ used for the inversion via the MCMC method displayed in Algorithm 4.1.

The Algorithm 4.1 requires, in step (i), sampling of the prior. This is accomplished by parameterizing the level set function in terms of the Karhunen-Loeve (KL) expansion associated to the prior covariance operator $\mathcal{C}$ (See Appendix 2, equation (7.1)). For the purpose of numerics, the infinite series of the KL expansion is truncated; theoretical results concerning the effect of this finite dimensional approximation on the posterior can be found in [20]. Upon discretization, the number of eigenvectors of $\mathcal{C}$ equals the dimensions of the discretized physical domain of the model problems (i.e. $N=6400$ in expression (7.2)). Once the eigendecomposition of $\mathcal{C}$ has been conducted, then sampling from the prior can be done simply by sampling an i.i.d set of random variables $\left\{\xi_{k}\right\}$ with $\xi_{1} \sim \mathcal{N}(0,1)$ and using it in (7.2). During the burn-in period (which here is taken to comprise $10^{4}$ iterations) of the MCMC method, we find it advantageous to freeze the higher KL modes and conduct the sampling only for the lower modes. After the aforementioned burn-in, the sampling is then carried out on the full set of KL modes represented on the given computational mesh. This freezing of modes udring the burn-in enables the MCMC method to quickly reach an "optimal" state where the samples of the level set function provide fields $\kappa(x)$ that are close to the truth. However, once 
this optimal state has been reached, it is essential to conduct the sampling on the full spectrum of $\mathrm{KL}$ modes to ensure that the MCMC chain mixes and properly represnts the posterior uncertainty. More precisely, if only the lowest modes are retained for the full chain, the MCMC may collapse into the optimal state but without mixing. Thus, while the lowest KL modes determine the main geometric structure of the underlying discontinuous field, the highest modes are essential for the proper mixing and thus the proper and efficient characterization of the posterior.

\subsection{Inverse Potential Problem}

In this experiment we generate synthetic data by solving (3.1), on a fine grid of size $240 \times 240$ with the "true" indicator function $\kappa^{\dagger}=\mathbb{I}_{D_{1}^{\dagger}}$ displayed in Figure 1 (top). The observation operator $\mathcal{O}=\left(\mathcal{O}_{1}, \ldots, \mathcal{O}_{64}\right)$ is defined in terms of 64 mollified Dirac deltas $\left\{\mathcal{O}_{j}\right\}_{j=1}^{64}$ centered at the measurement locations display as white squares along the boundary of the domain in Figure 1 (top). Each coordinate of the data is computed by means of (3.3) with $p$ from the solution of the PDE with the aforementioned true source term and by adding Gaussian noise $\eta_{j}$ with standard deviation of $10 \%$ of the size of the noise-free measurements (i.e. of $\mathcal{O}_{j}\left(\frac{\partial p}{\partial \nu}\right)$ ). We reiterate that, in order to avoid inverse crimes [37], we use a coarser grid of size $80 \times 80$ for the inversion via the MCMC method (Algorithm 4.1). The parameterization of $D_{1}$ in terms of the level set function is given by $D_{1}=\{x \in D \mid u(x)<0\}$ (i.e. by simply choosing $c_{0}=-\infty$ and $c_{1}=0$ in (2.3)).

For this example we consider a prior covariance $\mathcal{C}$ of the form presented in (2.17) for some choices of $L$ in the correlation function. We construct $\mathcal{C}$ directly from this correlation function and then we conduct the eigendecomposition needed for the KL expansion and thus for sampling the prior. In Figure 2 we display samples from the prior $\mathcal{N}(0, \mathcal{C})$ on the level set function $u$ (first, third and fifth rows) and the corresponding indicator function $\kappa=\mathbb{I}_{D_{1}}$ (second, fourth and sixth rows) for (from left to right) $L=0.1,0.15,0.2,0.3,0.4$. Different values of $L$ in (2.17) clearly result in substantial differences in the spatial correlation of the zero level set associated to the samples of the level set function. The spatial correlation of the zero level set funtion, under the prior, has significant effect on $\mathbb{I}_{D_{1}}$ which we use as the right-hand side (RHS) in problem (3.1) and whose solution, via expression (3.3), determines the likelihood (2.9). It then comes as no surprise that the posterior measure on the level set is also strong;y dependent on the choice of the prior via the parameter $L$. We explore this effect in the following paragraphs.

In Figure 3 we present the numerical results from different MCMC chains computed with different priors corresponding to the aforementioned choices of $L$. The MCMC mean of the level set function is displayed in the top row of Figure 3 for the choices (from left to right) $L=$ $0.1,0.15,0.2,0.3,0.4$. We reiterate that although the MCMC method provides the characterization of the posterior of the level set function, our primary aim is to identify the field $\kappa(x)=\mathbb{I}_{D_{1}}(x)$ that determines the RHS of (3.1) by means of conditioning the prior $\mathcal{N}(0, \mathcal{C})$ to noisy data from (3.3). A straightforward estimate of such field can be obtained by mapping, via the level set map (2.4), the posterior mean level set function denoted by $\bar{u}$ into the corresponding field $F(\bar{u}(x))=\bar{\kappa}(x)=$ 
$\mathbb{I}_{\bar{D}_{1}}(x)$ where $\bar{D}_{1}=\{x \in D \mid \bar{u}(x)<0\}$. We display $\bar{\kappa}(x)=\mathbb{I}_{\bar{D}_{1}}(x)$ in the top-middle row of Figure 3 along with the plot of the true field $\kappa^{\dagger}=\mathbb{I}_{D_{1}^{\dagger}}$ (right column) for comparison.

As mentioned earlier, we are additionally interested in the push-forward of the posterior measure of the level set function $u$ under the level set map (i.e. $\left(F^{*} \mu^{y}\right)(d u)$ ). We characterize $F^{*} \mu^{y}$ by mapping under $F$ our MCMC samples from $\mu^{y}$. In Figure 3 we present the mean (bottom-middle) and the variance (bottom) of $F^{*} \mu^{y}$. Figure 4 shows some posterior (MCMC) samples $u$ of the level set function (first, third and fifth rows) and the corresponding level set map $F(u)=\mathbb{I}_{D_{1}}$ with $D_{1}=\{x \in D \mid u(x)<0\}$ associated to these posterior samples (second, fourth and sixth rows).

The push-forward of the posterior measure under the level set map (i.e. $F^{*} \mu^{y}$ ) thus provides a probabilistic description of the inverse problem of identifying the true $\kappa^{\dagger}=\mathbb{I}_{D_{1}^{\dagger}}$. We can see from Figure 3 that, for some choices of $L$, the mean of $F^{*} \mu^{y}$ provides reasonable estimates of the truth. However, the main advantage of the Bayesian approach proposed here is that a measure of the uncertainty of such estimate is also obtained from $F^{*} \mu^{y}$. The variance (Figure 3 bottom), for example, is a measure of the uncertainty in the location of the interface between the two regions $D$ and $D \backslash D_{1}$.

The results displayed in Figure 3 show the strong effect that the selection of the prior has on the posterior measure $\mu^{y}$ and the corresponding pushforward measure $F^{*} \mu^{y}$. In particular, there seems to be a critical value $L=0.2$ above of which the corresponding posterior mean on $F^{*} \mu^{y}$ provides a reasonable identification of the true $\mathbb{I}_{D_{1}^{\dagger}}$ with relatively small variance. This critical value seems to be related to the size and the shape of the inclusions that determines the true region $D_{1}^{\dagger}$ (Figure 1 (top)). It is intuitive that posterior samples that result from very small spatial correlation cannot easily characterize these inclusions accurately unless the data is overwhelmingly informative. The lack of a proper characterization of the geometry from priors associated with small $L$ is also reflected with larger variance around the interface. It is then clear that the capability of the proposed level set Bayesian framework to properly identify a shape $D_{1}^{\dagger}$ (or alternatively its indicator function $\mathbb{I}_{D_{1}^{\dagger}}$ ) depends on properly incorporating, via the prior measure, a priori information on the regularity and spatial correlation of the unknown geometry of $D_{1}^{\dagger}$.

Since the selection of the prior has such a clear effect on the posterior, it comes as no surprise that it also affects the efficiency of the MCMC method as we now discuss. In the bottom-right panel of Figure 1 we show the autocorrelation function (ACF, see [12]) of the first KL mode of the level set function from different MCMC chains with different priors corresponding to our different choices of correlation length $L$ in (2.17). The tunable parameters in the pCN-MCMC method are fixed for these experiments. We recall from Figure 3 that larger values of $L$ result in a mean level set whose corresponding indicator function better captures the spatial structures form the truth and with smaller variance around the interface. However, the larger the value of $L$ the slower the decay of the ACF. From these ACF plots, we note that even for the apparent optimal value of $L=0.3$, our MCMC method produces samples that are highly correlated and thus very long chains may be needed in order to produce a reasonable number of uncorrelated samples needed for statistical 
analysis. For this particular choice of $L=0.3$ we have conducted 50 multiple MCMC chains of length $10^{6}$ (after burn-in period) initialized from random samples from the prior. In Figure 1 (bottom-left) we show the potential scale reduction factor (PSRF, see [11] for a defintion) computed from MCMC samples of the level set function (red-solid line) and the corresponding samples under $F$ (i.e. the $\mathbb{I}_{D_{1}}$ 's) (blue-dotted line) which corresponds to the RHS of (3.1). We observe that the PSRF goes below 1.1 after (often taken as an approximate indication of convergence [11]); thus the Gelman-Rubin diagnostic [11] based on the PSRF is passed for this selection of $L$. The generation of multiple independent MCMC chains that are statistically consistent opens up the possibility of using high-performance computing to enhance our capabilities of properly exploring the posterior. While we use a relatively small number of chains as a proof-of-concept, the MCMC chains are fully independent and so the computational cost of running multiple chains scales with the number of available processors.

The $5 \times 10^{7}$ samples that we obtained from the 50 MCMC chains are combined to provide a full characterization of the posterior $\mu^{y}$ on the level set and the corresponding push-forward $F^{*} \mu^{y}$ (i.e. The $\mathbb{I}_{D_{1}}$ 's computed from $D_{1}$ with posterior samples $u$ ). We reemphasize that our aim is the statistical identification of $\mathbb{I}_{D_{1} \dagger}$. Therefore, in order to obtain a quantity from the true $\mathbb{I}_{D_{1}^{\dagger}}$ against to which compare the computed push-forward of the level set posterior, we consider the Discrete Cosine Transform (DCT) of the true field $\mathbb{I}_{D}$. Other representations/expansions of the true field could be considered for the sake of assessing the uncertainty of our estimates with respect to the truth. In Figure 5 we show the prior and posterior densities of the first DCT coefficients of $\mathbb{I}_{D_{1}}$ where $D_{1}=\{x \in D \mid u(x)<0\}$ with $u$ from our MCMC samples (the vertical dotted line corresponds to the DCT coefficient of the true $\mathbb{I}_{D_{1}^{\dagger}}$ ). We can observe how the push forward posterior are concentrated around the true values. It is then clear how the data provide a strong conditioning on the first DCT coefficients of the discontinuous field that we aim at obtaining with our Bayesian level set approach.

While the main objective of our Bayesian methodology is to characterize the posterior, it is relevant to assess the accuracy of this methodology at approximating the truth $\kappa^{\dagger}$; doing so measures, simultanesouly, the information content in the data and the efficacy of the algorithm. To this end we define the following $L^{1}$-relative error:

$$
\mathcal{E}_{L^{1}}(\xi) \equiv \frac{\left\|\xi-\kappa^{\dagger}\right\|_{L^{1}(D)}}{\left\|\kappa^{\dagger}\right\|_{L^{1}(D)}}
$$

In Figure 6 (left) we plot $\mathcal{E}_{L^{1}}\left(F\left(\bar{u}_{n}\right)\right)$ which corresponds to the relative error with respect to the truth $\kappa^{\dagger}$, at the $n$th MCMC iteration, of the MCMC sample mean $\bar{u}_{n}$ under the map $F$. Figure 6 (middle) displays $\mathcal{E}_{L^{1}}\left(\bar{\kappa}_{n}\right)$, i.e. the error of the sample mean of the pushforward samples under $F$ (i.e. the mean of the samples $\kappa_{n}=F\left(u_{n}\right)$ ). Finally, in Figure 6 (right) we show $\mathcal{E}_{L^{1}}\left(F\left(u_{n}\right)\right)$, the error of the pushforward under $F$ of the $n$th MCMC sample. We can clearly appreciate that the most accurate results corresponds to $L=0.3$ and $L=0.4$ which are, in turn, the cases with less uncertainty in terms of the variance (see Figure 3 bottom row). The larger size of the errors in the rightmost panel 
is a reflection of the uncertainty in the reconstruction, and the posterior variance in the estimates.
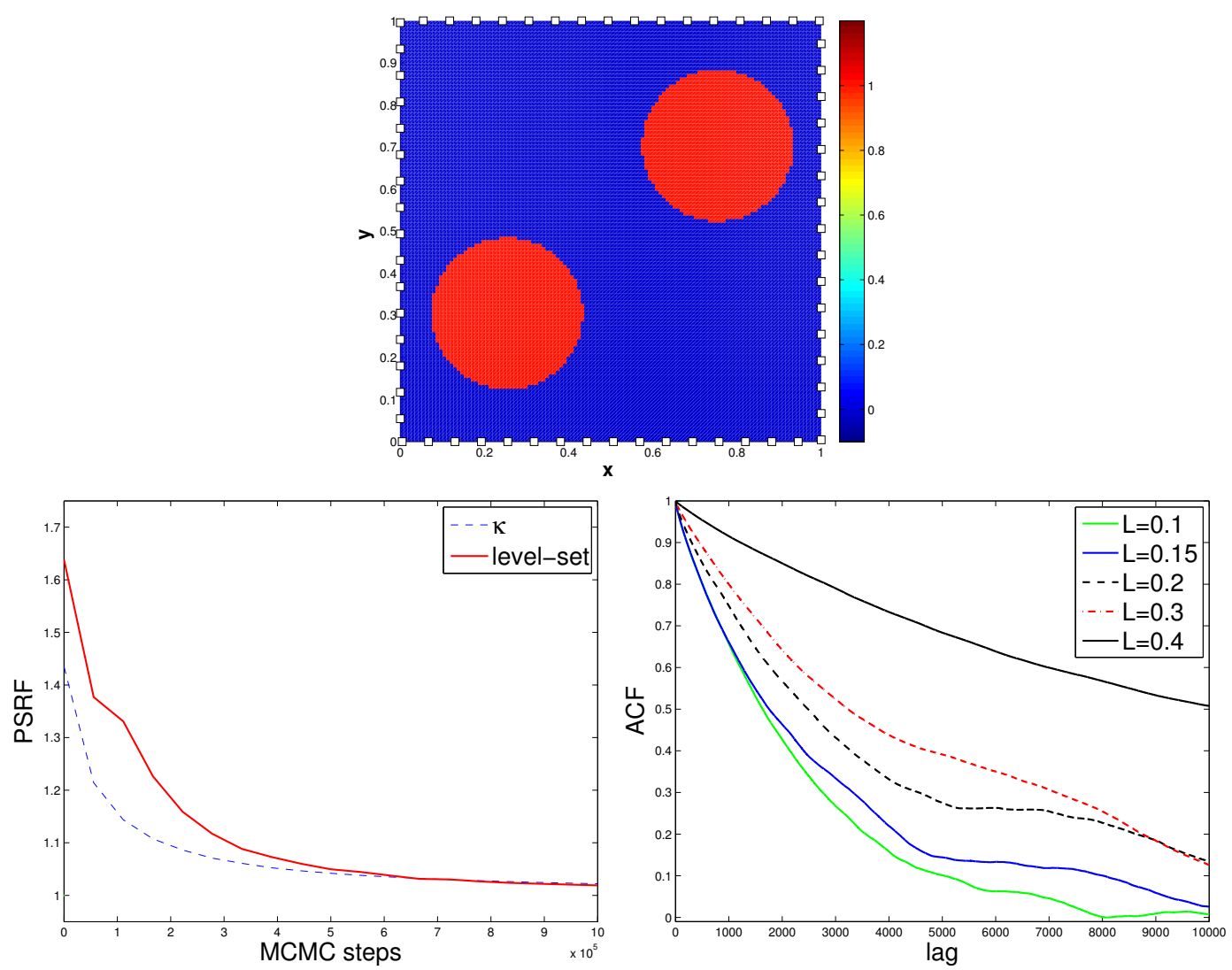

Figure 1: Inverse Potential. Top: True source term $\kappa^{\dagger}=\mathbb{I}_{D_{1}^{\dagger}}$ of eq. (3.1). Bottom-left: PSRF from multiple chains with $L=0.3$ in (2.17). The PSRF is computed from level-set samples (solid red line) as well as the corresponding $\kappa=\mathbb{I}_{D_{1}}$ (blue dotted line). Bottom-right: ACF of first KL mode of the level set function from single-chain MCMC with different choices of $L$. 

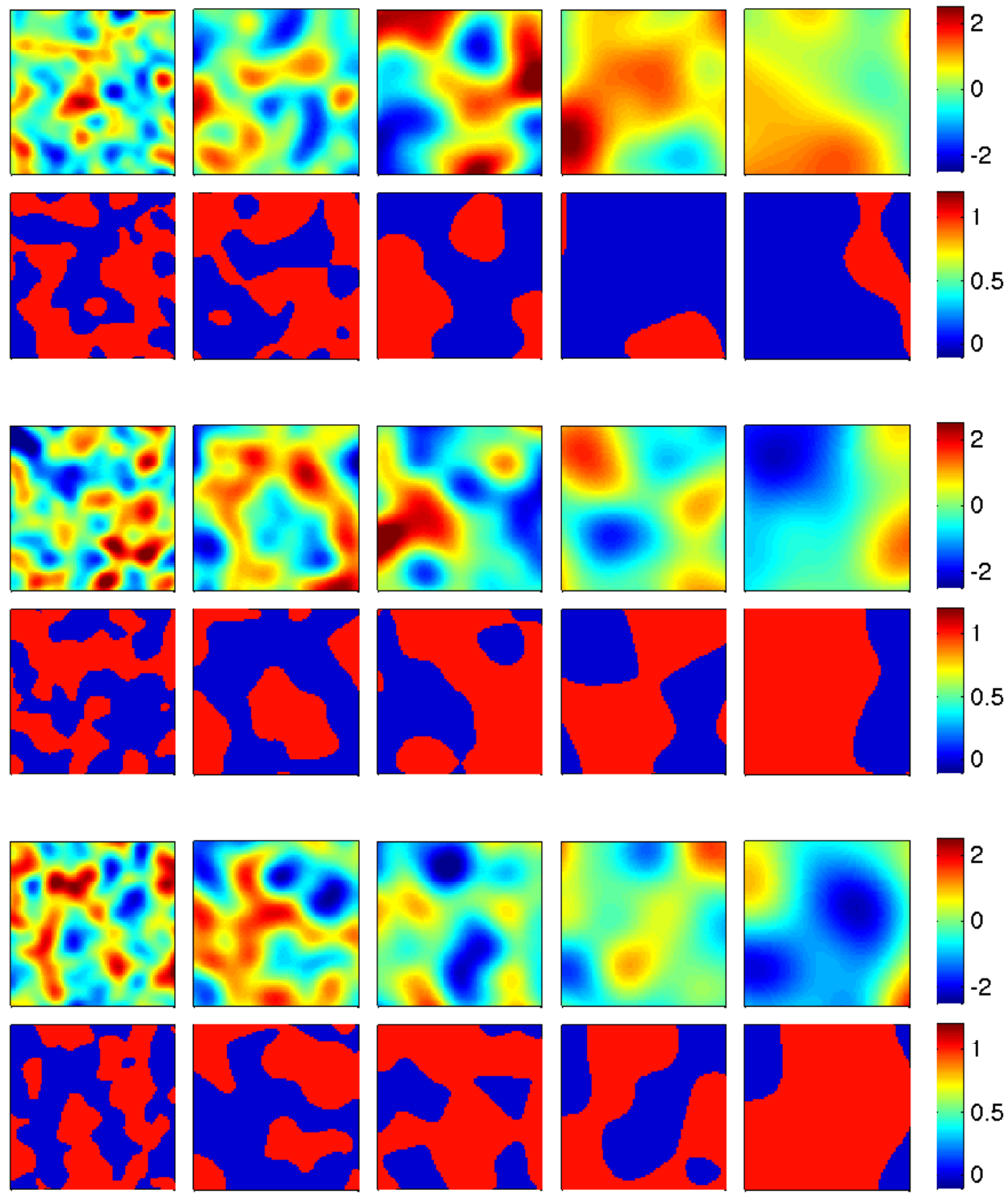

Figure 2: Inverse Potential. Samples from the prior on the level set function $u$ (first, third and fifth rows) for (from left to right) $L=0.1,0.15,0.2,0.3,0.4$. Corresponding $\mathbb{I}_{D_{1}}$ with $D_{1}=\{x \in$ $D \mid u(x)<0\}$ (second, fourth and sixth rows) associated to each of these samples from the level set function. 

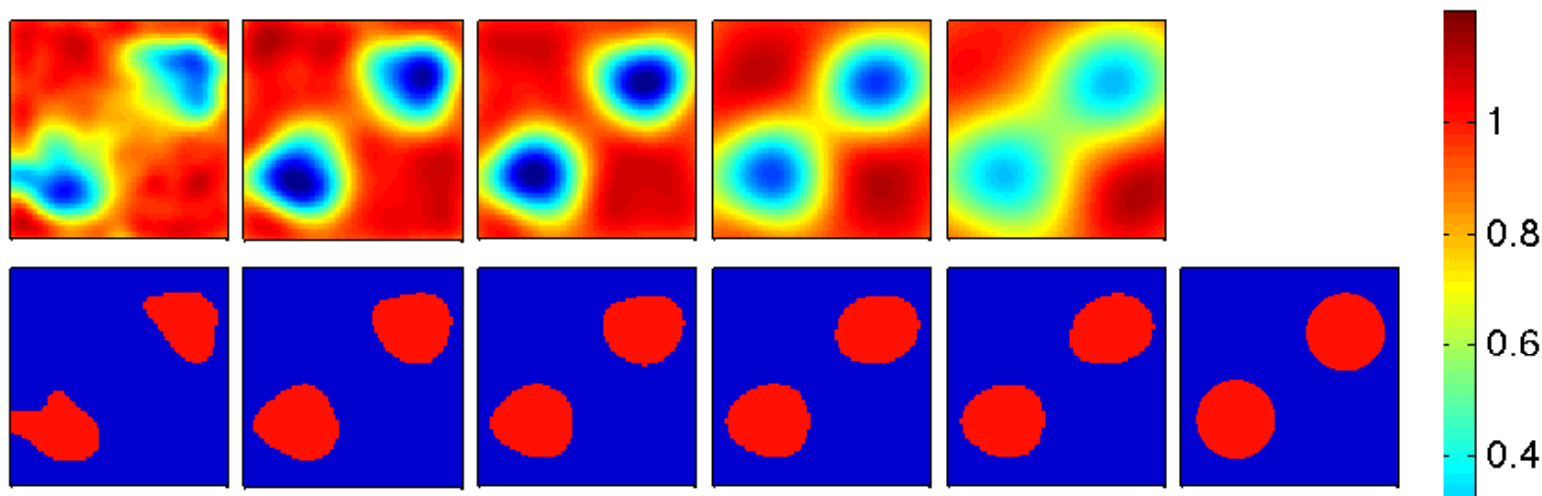

0.8
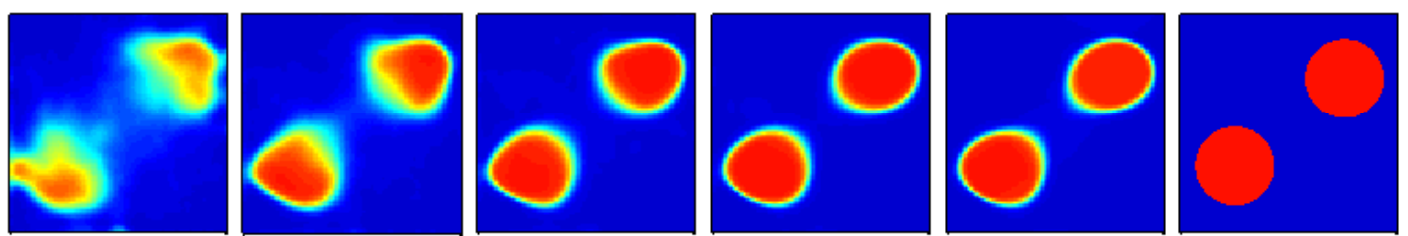

$-0.6$
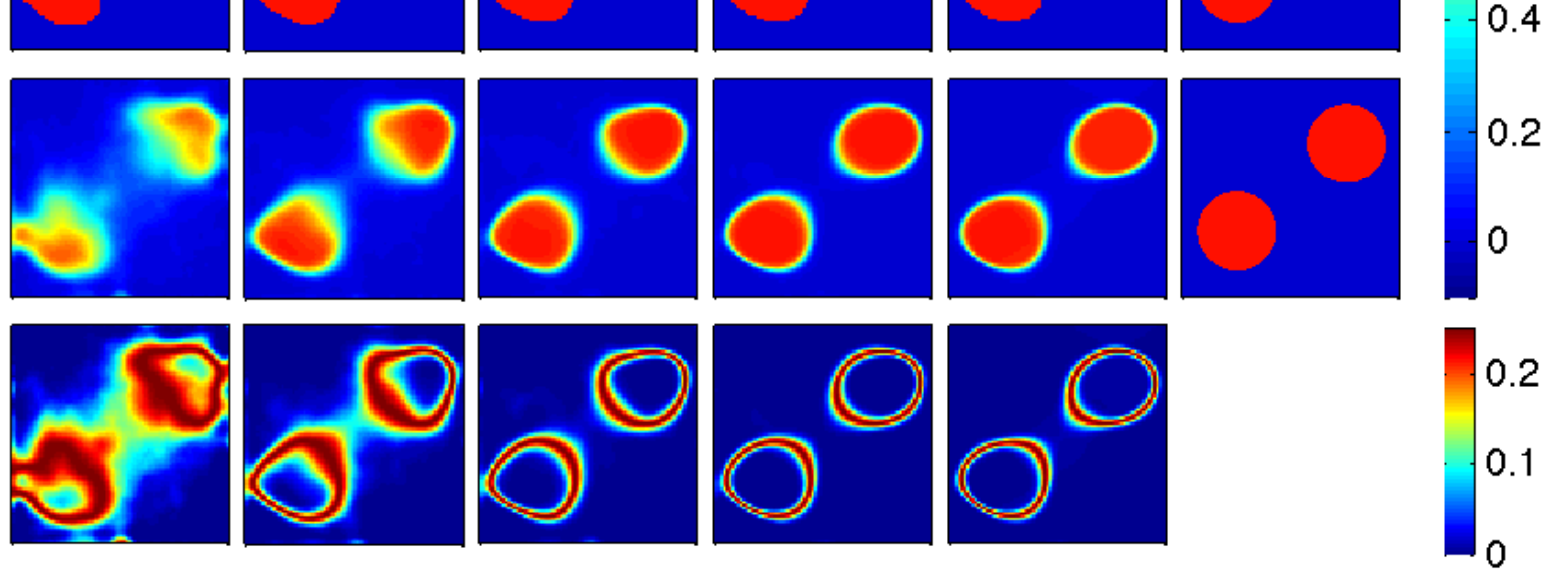

Figure 3: Inverse Potential. MCMC results for (from left to right) $L=0.1,0.15,0.2,0.3,0.4$ in the eq. (2.17). Top: Posterior mean level set function $\bar{u}$ (computed via MCMC). Top-middle: Plot of $\mathbb{I}_{\bar{D}_{1}}$ with $\bar{D}_{1}=\{x \in D \mid \bar{u}(x)<0\}$ (the truth $\mathbb{I}_{D_{1}^{\dagger}}$ is presented in the right column). Bottom-middle: Mean of $\mathbb{I}_{D_{1}}$ where $D_{1}=\{x \in D \mid u(x)<0\}$ and $u$ 's are the posterior MCMC samples (the truth is presented in the right column). Bottom: Variance of $\mathbb{I}_{D_{1}}$ where $D_{1}=\{x \in D \mid u(x)<0\}$ and $u$ 's are the posterior MCMC samples 

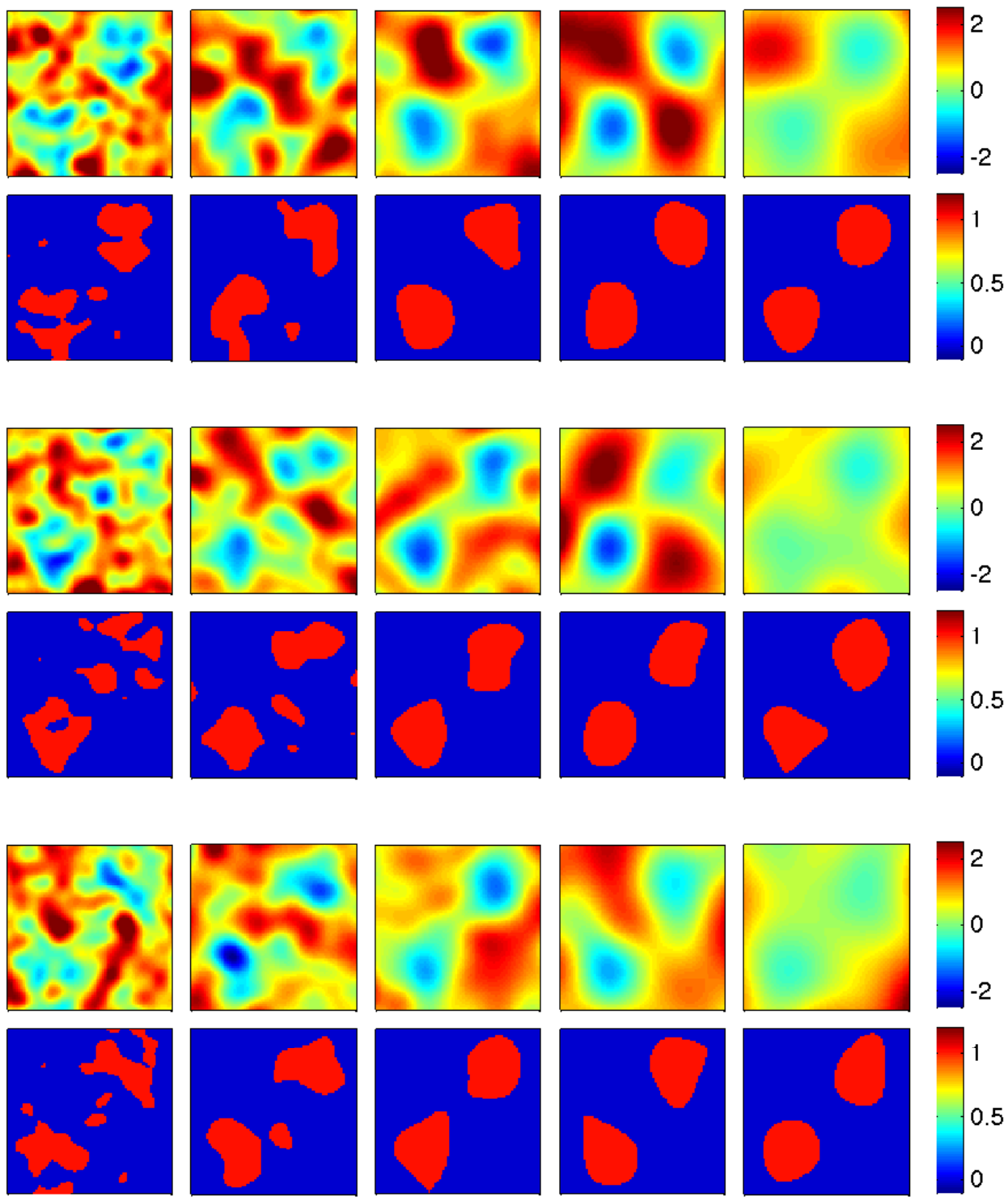

Figure 4: Inverse Potential. Samples from the posterior on the level set $u$ (first, third and fifth rows) for (from left to right) $L=0.1,0.15,0.2,0.3,0.4$. Corresponding $\mathbb{I}_{D_{1}}$ where $D_{1}=\{x \in D \mid u(x)<$ $0\}$ (second, fourth and sixth rows) associated to each of these samples from the level set function. 

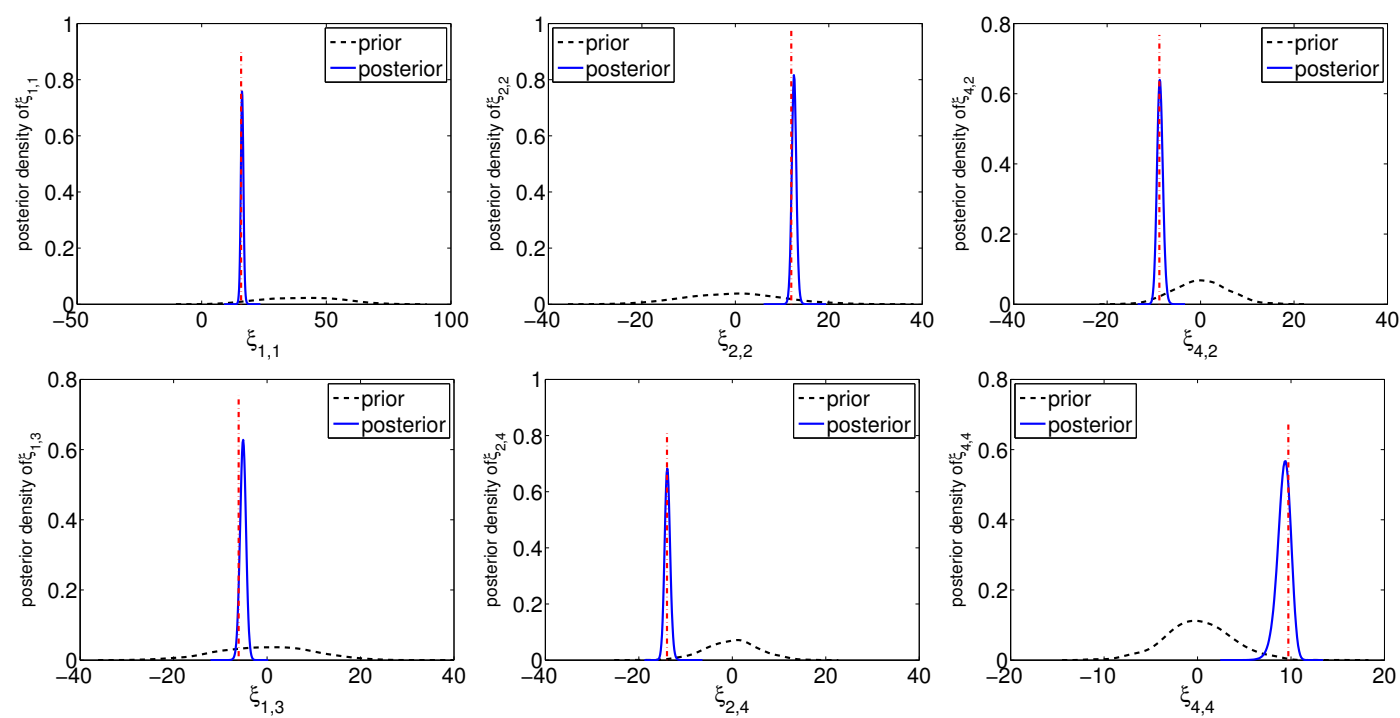

Figure 5: Inverse Potential. Densities of the prior and posterior of various DCT coefficients of the $\mathbb{I}_{D_{1}}$ where $D_{1}=\{x \in D \mid u(x)<0\}$ obtained from MCMC samples on the level set $u$ for $L=0.3$ (vertical dotted line indicates the truth).
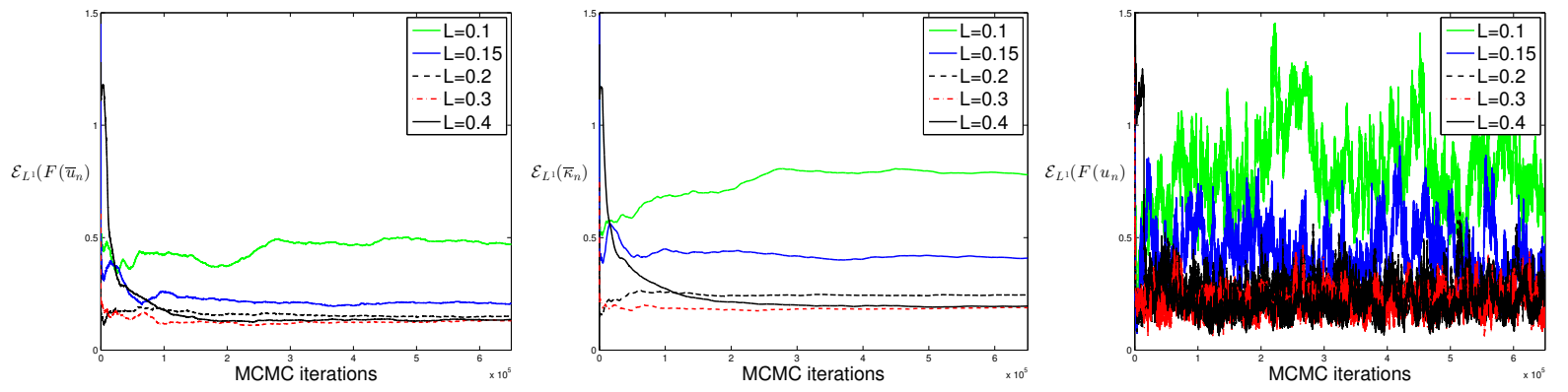

Figure 6: Inverse Potential. $L^{1}(D)$ relative errors with respect to the truth for different choices of $L$. Left: $\mathcal{E}_{L^{1}}\left(F\left(\bar{u}_{n}\right)\right)$. Middle: $\mathcal{E}_{L^{1}}\left(\bar{\kappa}_{n}\right)$. Right: $\mathcal{E}_{L^{1}}\left(F\left(u_{n}\right)\right)$ 


\subsection{Structural Geology: Channel Model}

In this section we consider the inverse problem discussed in subsection 3.2. We consider the Darcy model (3.4) but with a more realistic set of boundary conditions that consist of a mixed Neumann and Dirichlet conditions. For the concrete set of boundary conditions as well as the right-hand-side we use for the present example we refer the reader to [31, Section 4]. This flow model, initially used in the seminal paper of [17], has been used as a benchmark for inverse problems in [31, 28, 30]. While the mathematical analysis of subsection is 3.2 conducted on a model with Dirichlet boundary conditions, in order to streamline the presentation, the corresponding extension to the case of mixed boundary conditions can be carried out with similar techniques.

We recall that the aim is to estimate the interface between regions $D_{i}$ of different structural geology which result in a discontinuous permeability $\kappa$ of the form (2.2). In order to generate synthetic data, we consider a true $\kappa^{\dagger}(x)=\sum_{i=1}^{3} \kappa_{i} \mathbb{I}_{D_{i}^{\dagger}}$ with prescribed (known) values of $\kappa_{1}=7, \kappa_{2}=50$ and $\kappa_{3}=500$. This permeability, whose plot is displayed in Figure 7 (top), is used in (3.4) to generate synthetic data collected from interior measurement locations (white squares in Figure 7). The estimation of $\kappa$ is conducted given observations of the solution of the Darcy model (3.4). To be concrete, the observation operator $\mathcal{O}=\left(\mathcal{O}_{1}, \ldots, \mathcal{O}_{25}\right)$ is defined in terms of 25 mollified Dirac deltas $\left\{\mathcal{O}_{j}\right\}_{j=1}^{25}$ centered at the aforementioned measurement locations and acting on the solution $p$ of the Darcy flow model. For the generation of synthetic data we use a grid of $160 \times 160$ which, in order to avoid inverse crimes [37], is finer than the one used for the inversion $(80 \times 80)$. As before, observations are corrupted with Gaussian noise proportional to the size of the noise-free observations $\left(\mathcal{O}_{j}(p)\right.$ in this case).

For the estimation of $\kappa$ with the proposed Bayesian framework we assume that knowledge of three nested regions is available with the permeability values $\left\{\kappa_{i}\right\}_{i=1}^{3}$ that we use to define the true $\kappa^{\dagger}$. Again, we are interested in the realistic case where the rock types of the formation are known from geologic data but the location of the interface between these rocks is uncertain. In other words, the unknowns are the geologic facies $D_{i}$ that we parameterize in terms of a level set function, i.e. $D_{i}=\left\{x \in D \mid c_{i-1} \leq u(x)<c_{i}\right\}$ with $c_{0}=-\infty, c_{1}=0, c_{2}=1, c_{3}=\infty$. Similar to the previous example, we use a prior of the form (2.17) for the level set function. In Figure 8 we display samples from the prior on the level set function (first, third and fifth rows) and the corresponding permeability mapping under the level set map (2.4) $F(u)(x)=\kappa(x)=\sum_{i=1}^{3} \kappa_{i} \mathbb{I}_{D_{i}}$ (second, fourth and sixth rows) for (from left to right) $L=0.2,0.3,0.35,0.4,0.5$. As before, we note that the spatial correlation of the covariance function has a significant effect on the spatial correlation of the interface between the regions that define the interface between the geologic facies (alternatively, the discontinuities of $\kappa$ ). Longer values of $L$ provide $\kappa$ 's that seem more visually consistent with the truth. The results from Figure 9 show MCMC results from experiments with different priors corresponding to the aforementioned choices of $L$. The posterior mean level set function $\bar{u}$ is displayed in the top row of Figure 9. The corresponding mapping under the level set function $\bar{\kappa} \equiv \sum_{i=1}^{3} \kappa_{i} \mathbb{I}_{\bar{D}_{i}}$ (with $\bar{D}_{i}=\left\{x \in D \mid c_{i-1} \leq \bar{u}(x)<c_{i}\right\}$ ) is shown in the top-middle. 
Similar to our discussion of the preceding subsection, for the present example we are interested in the push-forward of the posterior $\mu^{y}$ under the level set map $F$. More precisely, $F^{*} \mu^{y}$ provides a probability description of the solution to the inverse problem of finding the permeability given observations from the Darcy flow model. In Figure 9 we present the mean (bottom-middle) and the variance (bottom) of $F\left(\mu^{y}\right)$ characterized by posterior samples on the level set function mapped under $F$. In other words, these are the mean and variance from the $\kappa$ 's obtained from the MCMC samples of the level / set function. As in the previous example, there is a critical value of $L=0.3$ below of which the posterior estimates cannot accurately identify the main spatial features of $\kappa^{\dagger}$. Figure 10 shows posterior samples of the level set function (first, third and fifth rows) and the corresponding $\kappa$ (second, fourth and sixth rows). The posterior samples, for values of $L$ above the critical value $L=0.3$, capture the main spatial features from the truth. There is, however, substantial uncertainty in the location of the interfaces. Our results offer evidence that this uncertainty can be properly captured with our level set Bayesian framework. Statistical measures of $F^{*} \mu^{y}$ (i.e. the posterior permeability measure on $\kappa$ ) is essential in practice. The proper quantification of the uncertainty in the unknown geologic facies is vital for the proper assessment of the environmental impact in applications such as $\mathrm{CO}_{2}$ capture and storage, nuclear waste disposal and enhanced oil recovery.

In Figure 7 (bottom-right) we show the ACF of the first KL mode of level set function from different MCMC chains corresponding to different priors defined by the choices of $L$ indicated previously. In contrast to the previous example, here we cannot appreciate substantial differences in the efficiency of the chain with respect to the selected values of $L$. However, we note that ACF exhibits a slow decay and thus long chains and/or multiple chains are need to properly explore the posterior. For the choice of $L=0.4$ we consider 50 multiple MCMC chains. Our MCMC chains pass the Gelman-Rubin test [11] as we can note from Figure 7 (bottom-left) where we show the PSRF computed from MCMC samples of the level set function $u$ (red-solid line) and the corresponding mapping, under the level set map, into the permeabilities $\kappa$ (blue-dotted line). As indicated earlier, we may potentially increase the number of multiple chains and thus the number of uncorrelated samples form the posterior.

Figure 11 shows the prior and posterior densities of the first DCT coefficients on the $\kappa$ obtained from the MCMC samples of the level set function (the vertical dotted line corresponds to the DCT coefficient of the truth $\kappa^{\dagger}$ ). For some of these modes we clearly see that the posterior is concentrated around the truth. However, for the mode $\xi_{4,4}$ we note that the posterior is quite close to the prior indicating that the data have not informed this mode in any significant way.

Finally, in Figure 12 we display relative errors $\mathcal{E}_{L^{1}}\left(F\left(\bar{u}_{n}\right)\right)\left(\right.$ left), $\mathcal{E}_{L^{1}}\left(\bar{\kappa}_{n}\right)$ (middle) and $\mathcal{E}_{L^{1}}\left(F\left(u_{n}\right)\right)$ (right) with $\mathcal{E}_{L^{1}}$ as defined in (4.1). Accurate approximations are found for $L=0.3,0.35,0.4$. As in Figure 6, the larger size of the errors in the rightmost panel is a reflection of the uncertainty in the reconstruction, and the posterior variance in the estimates. 


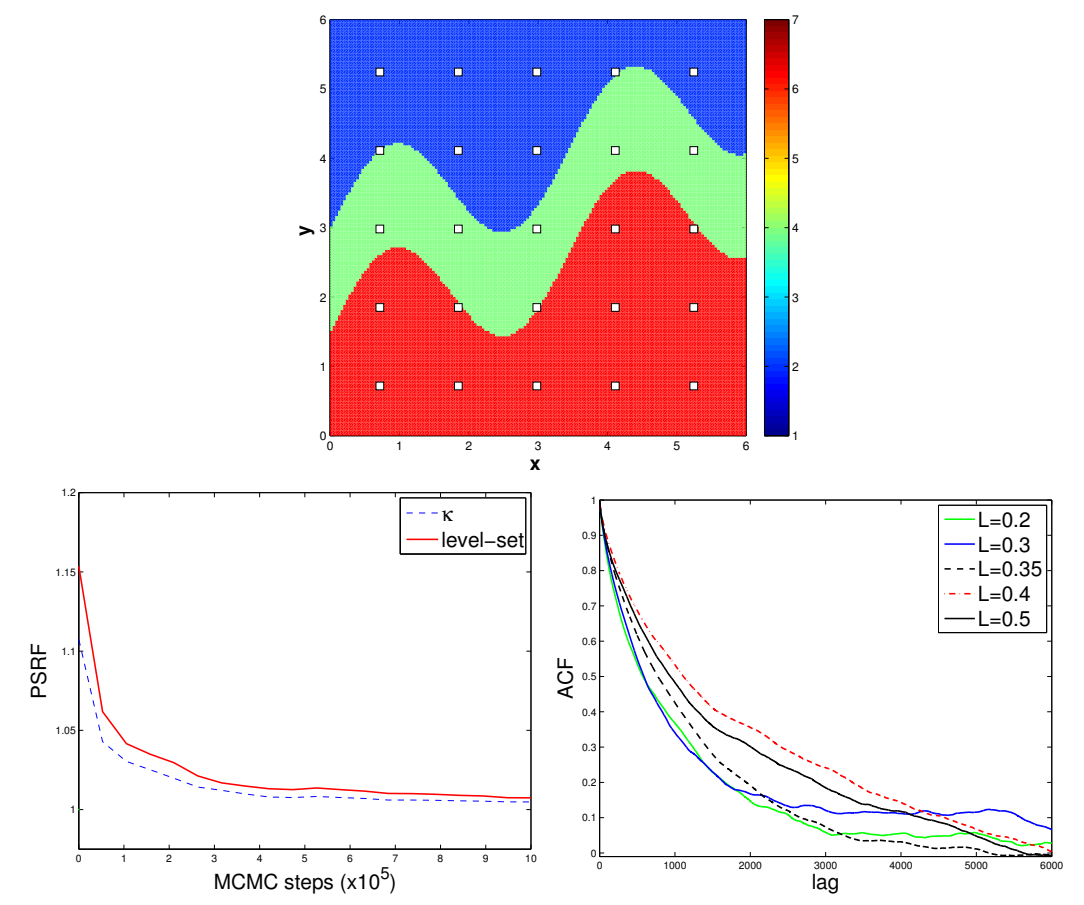

Figure 7: Identification of structural geology (channel model). Top: True $\kappa$ in eq. (3.4). Bottom-left: PSRF from multiple chains with $L=0.4$ in (2.16). Bottom-right: ACF of first KL mode of the level set function from single-chain MCMC with different choices of $L$.

\subsection{Structural Geology: Layer Model}

In this experiment we consider the groundwater model (3.4) with the same domain and measurement configurations from the preceding subsection. However, for this case we define the true permeability $\kappa^{\dagger}$ displayed in Figure 13 (top). The permeability values are as before. The generation of synthetic data is conducted as described in the preceding subsection. For this example we consider the Gaussian prior on the level set defined by (2.16). Since for this case the operator $\mathcal{C}$ is diagonalisable by cosine functions, we use the fast Fourier transform to sample from the corresponding Gaussian measure $\mathcal{N}(0, \mathcal{C})$ required by the $\mathrm{pCN}-\mathrm{MCMC}$ algorithm.

The tunable parameter $\alpha$ in the covariance operator (2.16) determines the regularity of the corresponding samples of the Gaussian prior (see for example [49]). Indeed, in Figure 14 we show samples from the prior on the level set function (first, third and fifth rows) and the corresponding $\kappa$ (second, fourth and sixth rows) for (from left to right) $\alpha=1.5,2.0,2.5,3.0,3.5$. Indeed, changes in $\alpha$ have a dramatic effect on the regularity of the interface between the different regions. We therefore expect strong effect on the resulting posterior on the level set and thus on the permeability.

In Figure 15 we display numerical results from MCMC chains with different priors corresponding to (from left to right) $\alpha=1.5,2.0,2.5,3.0,3.5$. In Figure 15 we present the MCMC mean of the level set function.The corresponding $\kappa$ is shown in the top-middle of Figure 15. In this figure we 

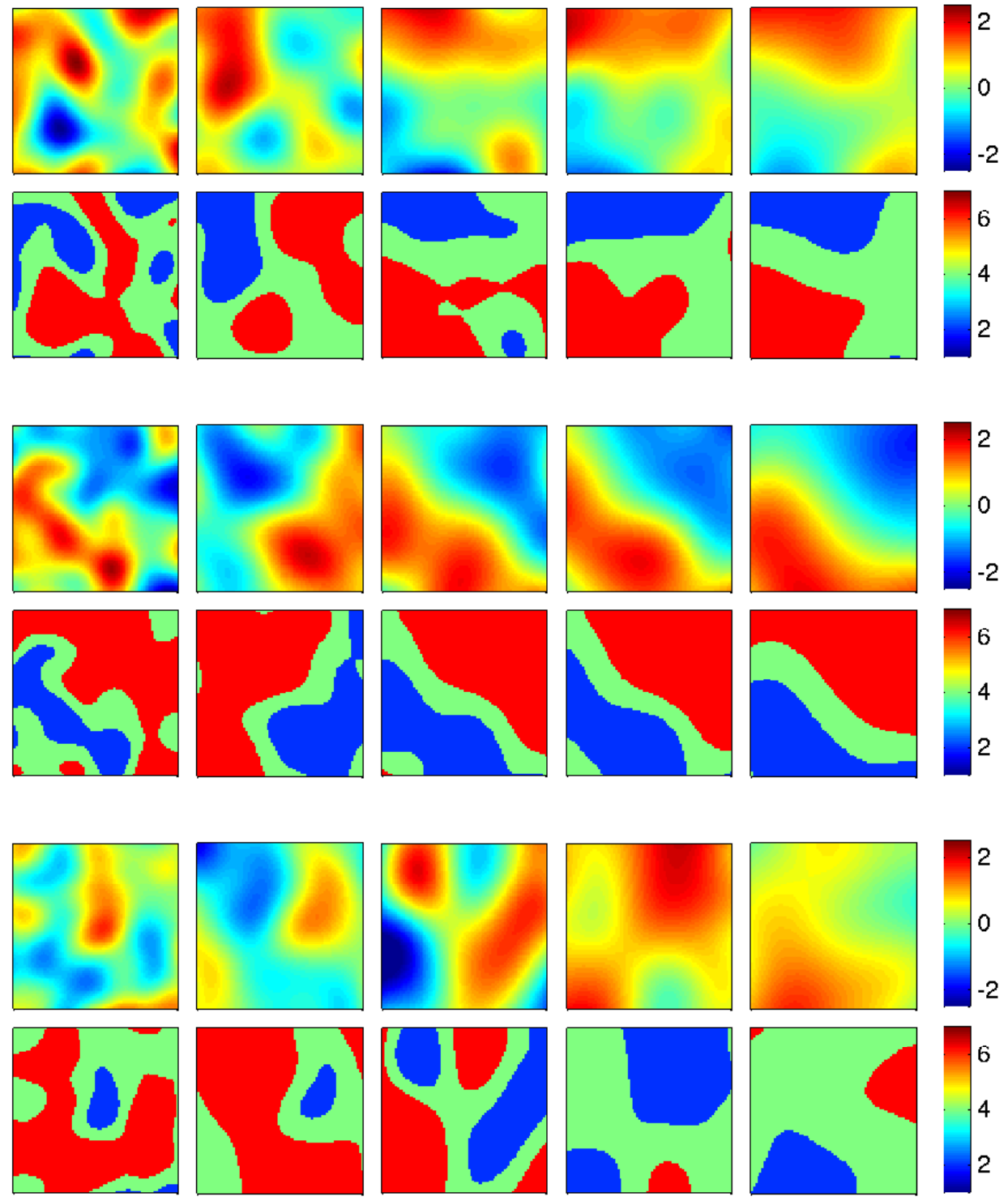

Figure 8: Identification of structural geology (channel model). Samples from the prior on the level set (first, third and fifth rows) for (from left to right) $L=0.2,0.3,0.35,0.4,0.5$. Pushforward onto $\kappa$ (second, fourth and sixth rows) associated to each of these samples from the level set function. 

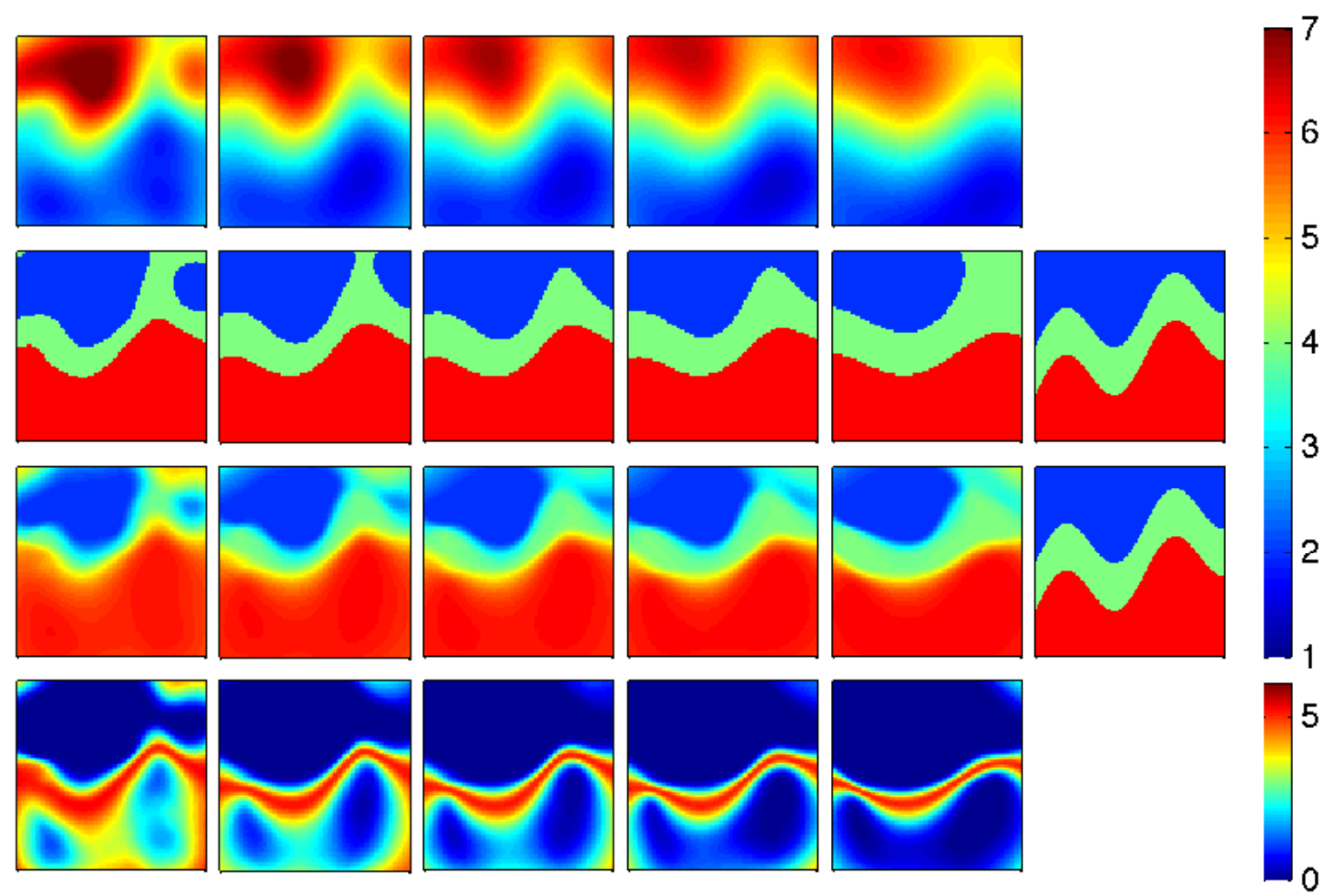

Figure 9: Identification of structural geology (channel model). MCMC results for (from left to right) $L=0.2,0.3,0.35,0.4,0.5$ in the eq. (2.16). Top: MCMC mean of the level set function. Top-middle: $\kappa$ associated to the mean of the level set function (true $\kappa$ is displayed in the last column). Bottommiddle: Mean of the $\kappa$. Bottom: Variance of $\kappa$ 

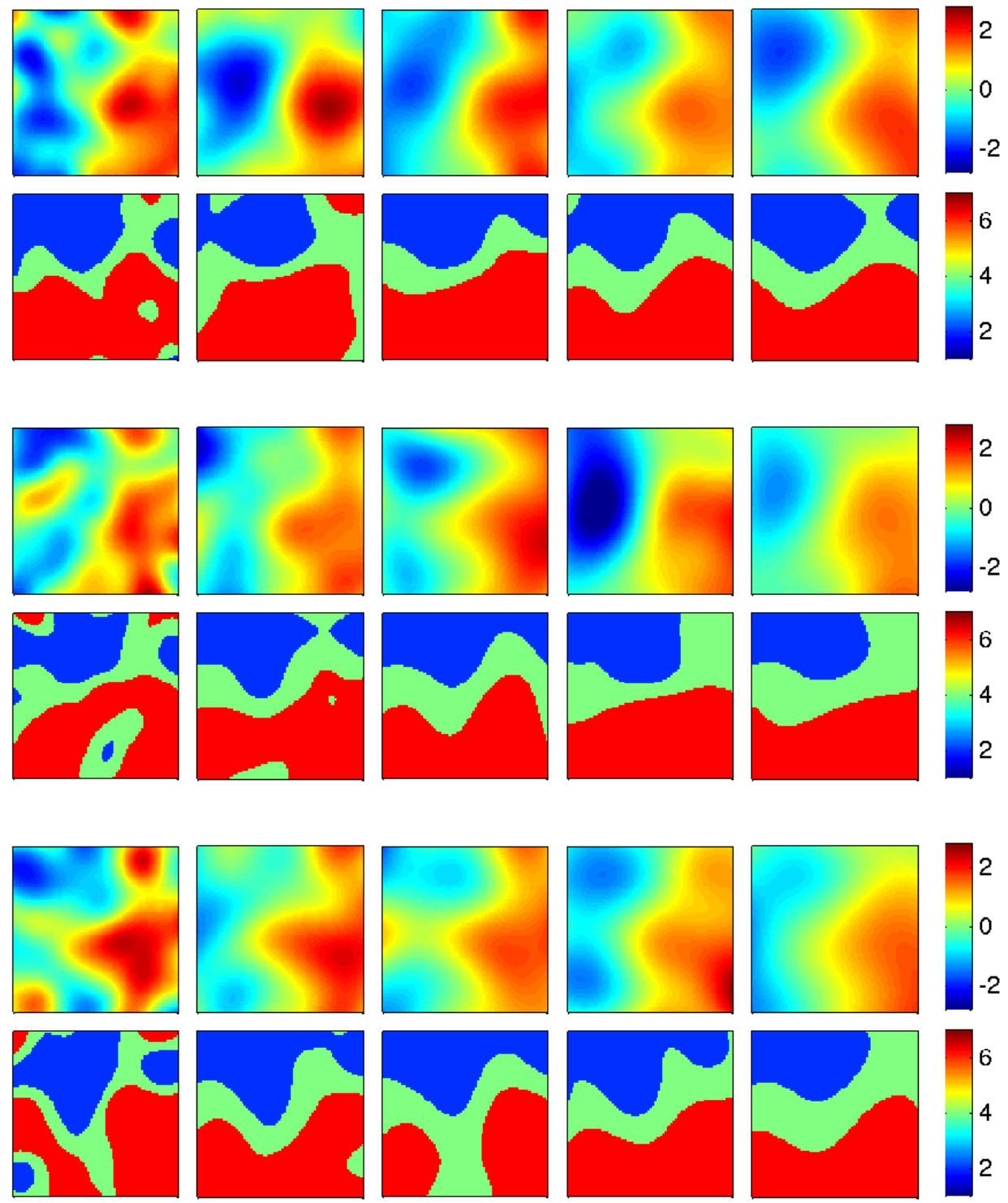

Figure 10: Identification of structural geology (channel model). Samples from the posterior on the level set (first, third and fifth rows) for (from left to right) $L=0.2,0.3,0.35,0.4,0.5 \cdot \log (\kappa)$ (second, fourth and sixth rows) associated to each of these samples from the level set function. 

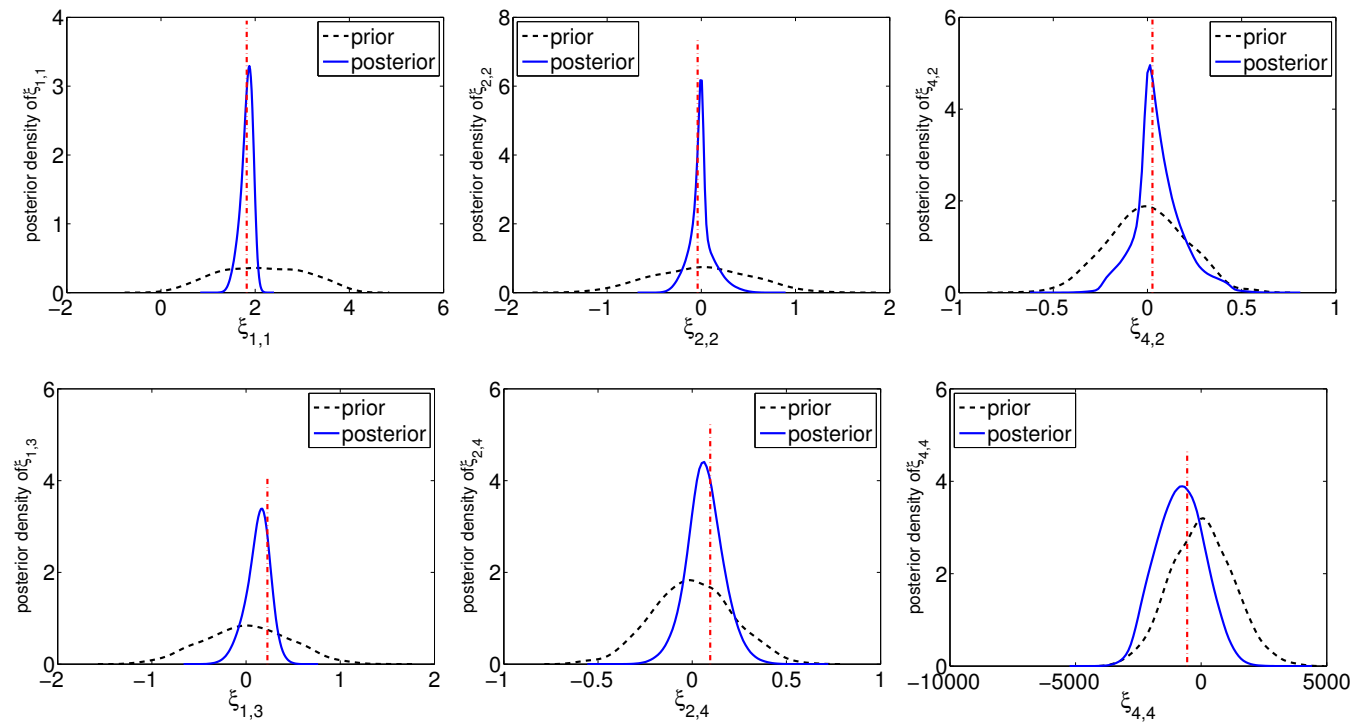

Figure 11: Identification of structural geology (channel model). Densities of the prior and posterior of some DCT coefficients of the $\kappa$ 's obtained from MCMC samples on the level set for $L=0.4$ (vertical dotted line indicates the truth).
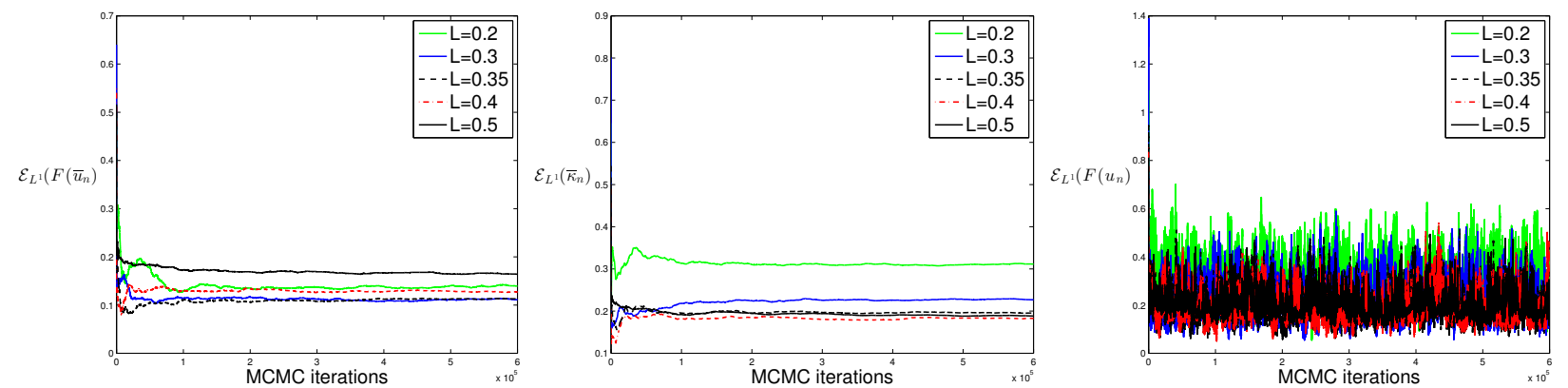

Figure 12: Identification of structural geology (channel model). $L^{1}(D)$ relative errors with respect to the truth for different choices of $L$. Left: $\mathcal{E}_{L^{1}}\left(F\left(\bar{u}_{n}\right)\right)$. Middle: $\mathcal{E}_{L^{1}}\left(\bar{\kappa}_{n}\right)$. Right: $\mathcal{E}_{L^{1}}\left(F\left(u_{n}\right)\right)$ 
additionally display the mean (bottom-middle) and the variance (bottom) of the $\kappa$ 's obtained from the MCMC samples of the level set function. Above a critical value $\alpha=2.5$ we obtain a reasonable identification of the layer permeability with a small uncertainty (quantified by the variance). Figure 16 shows posterior (MCMC) samples of the level set function (first, third and fifth rows) and the corresponding $\kappa$ (second, fourth and sixth rows) for the aforementioned choices of $\alpha$.

Figure 13 (bottom-right) shows the ACF of the first KL mode of level set function from MCMC experiments with different priors with $\alpha$ 's as before. The efficiency of the MCMC chain does not seem to vary significantly for the values above the critical value of $\alpha$. However, as in the previous examples a slow decay in the ACF is obtained. An experiment using 50 multiple MCMC chains initialized randomly from the prior reveals that the Gelman-Rubin diagnostic test [11] is passed for $\alpha=2.5$ as we can observe from Figure 13 (bottom-left) where we the display PSRF from MCMC samples of the level set function (red-solid line) and the corresponding mapping into the $\kappa$ (bluedotted line). In Figure 17 we show the prior and posterior densities of the DCT coefficients on the $\kappa$ obtained from the MCMC samples of the level set function (the vertical dotted line corresponds to the truth DCT coefficient). We see clearly that the DCT coefficients are substantially informed by the data although the spread around the truth confirms the variability in the location of the interface between the layers that we can ascertain from the posterior samples (see Figure 16).

In Figure 18 we present the relative errors with respect to the truth $\mathcal{E}_{L^{1}}\left(F\left(\bar{u}_{n}\right)\right)$ (left), $\mathcal{E}_{L^{1}}\left(\bar{\kappa}_{n}\right)$ (middle) and $\mathcal{E}_{L^{1}}\left(F\left(u_{n}\right)\right)$ (right). We note that $\alpha=3.5$ provides the most accurate approximation of the truth. As in Figure 6, the larger size of the errors in the rightmost panel is a reflection of the uncertainty in the reconstruction, and the posterior variance in the estimates.

\section{Conclusions}

The primary contributions of this paper are:

- We have formulated inverse problems for interfaces, within the Bayesian framework, using a level set approach.

- This framework leads to a well-posedness of the level set approach, something that is hard to obtain in the context of classical regularization techniques for level set inversion of interfaces.

- The framework also leads to the use of state-of-the-art function-space MCMC methods for sampling of the posterior distribution on the level set function. An explicit motion law for the interfaces is not needed: the MCMC accept-reject mechanism implicitly moves them.

- We have studied two examples: inverse source reconstruction and an inverse conductivity problem. In both cases we have demonstrated that, with appropriate choice of priors, the abstract theory applies. We have also highlighted the behavior of the algorithms when applied to these problems. 


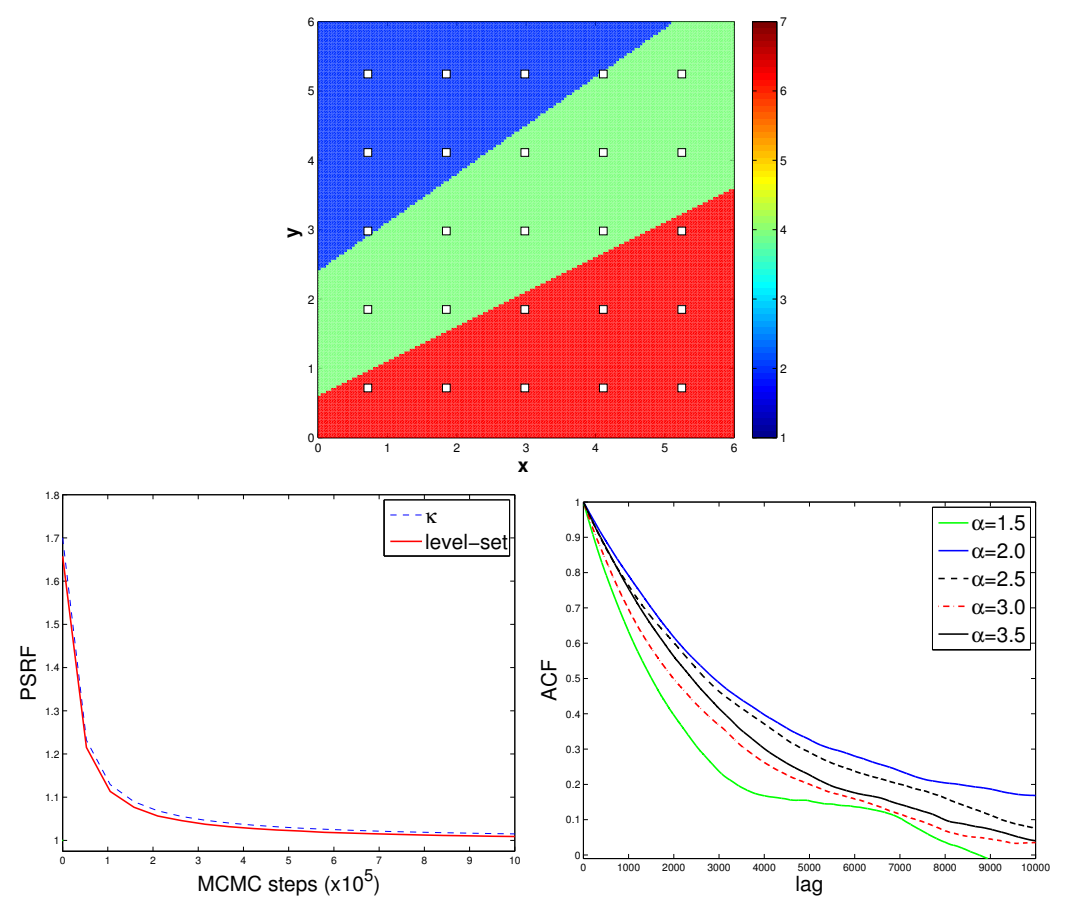

Figure 13: Identification of structural geology (layer model). Top: True $\kappa$ in eq. (3.4). Bottom-left: PSRF from multiple chains with $\alpha=2.5$ in (2.17). Bottom-right: ACF of first KL mode of the level set function from single-chain single-chain MCMC with different choices of $\alpha$.

- The fact that no explicit level set equation is required helps to reduce potential issues arising in level set inversion, such as reinitialization. In most computational level set approaches [16], the motion of the interface by means of the standard level set equation often produces level set functions that are quite flat. For the mean curvature flow problem, such flattening phenomena was observed early in the history of level set evolution in [26] where the surface evolution starts from a "figure eight" shaped initial surface; in addition it has been shown to happen even if the initial surface is smooth [4]. This causes stagnation as the interface then moves slowly. Ad-hoc approaches, such as redistancing/reinitializing the level set function with a signed distance function, are then employed to restore the motion of the interface. In the proposed computational framework, not only does the MCMC accept-reject mechanism induce the motion of the interface, but it does so in a way that avoids creating flat level set functions underlying the permeability. Indeed, we note that the posterior samples of the level set function inherit the same properties from the ones of the prior. In particular, the probability of obtaining a level set function which takes any given value on a set of positive measure is zero under the posterior, as it is under the prior. This fact promotes very desirable, and automatic, algorithmic robustness.

Natural directions for future research include the following: 

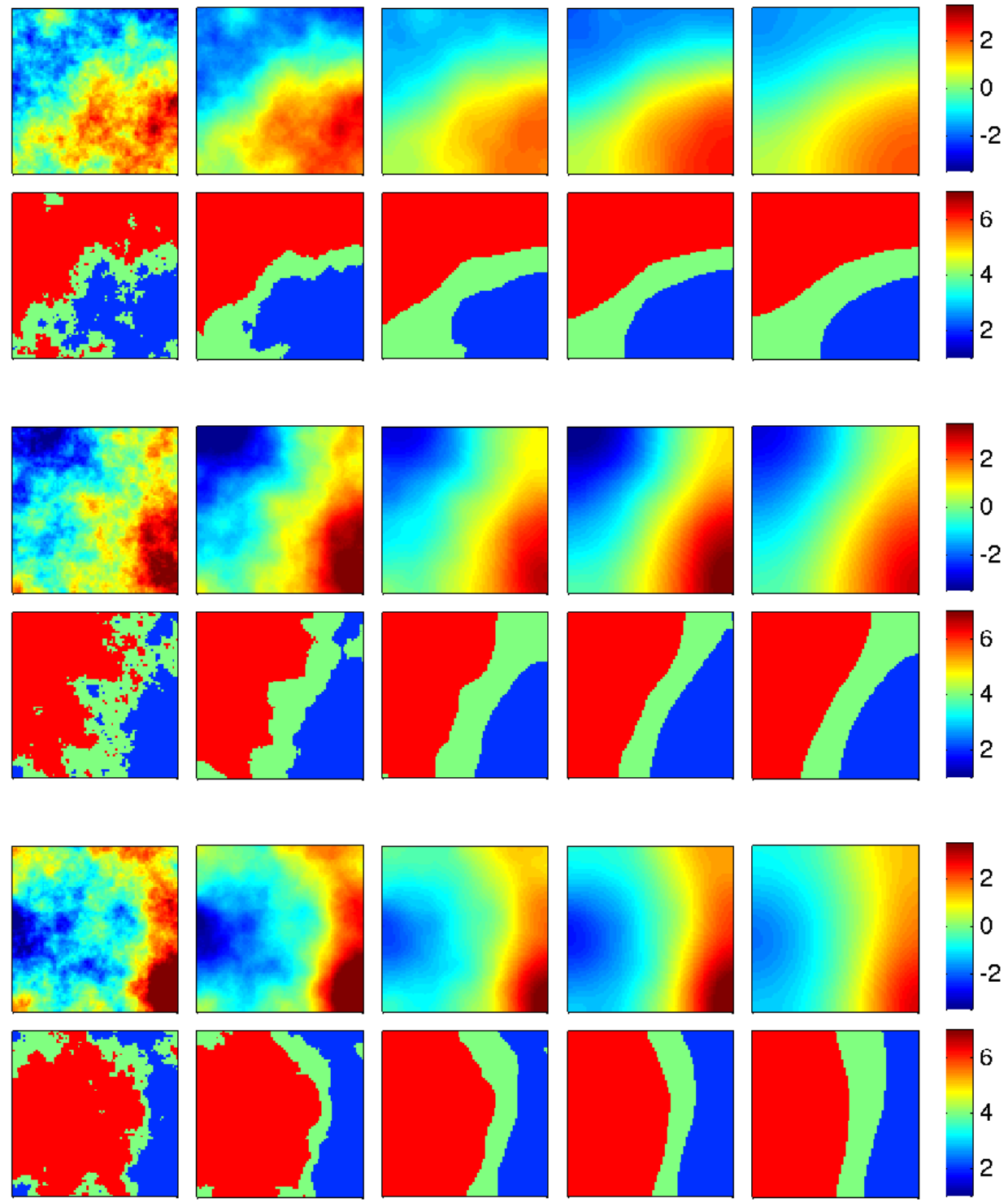

Figure 14: Identification of structural geology (layer model). Samples from the prior on the level set (first, third and fifth rows) for (from left to right) $\alpha=1.5,2.0,2.5,3.0,3.5$ in (2.17). $\kappa$ (second, fourth and sixth rows) associated to each of these samples from the level set function. 

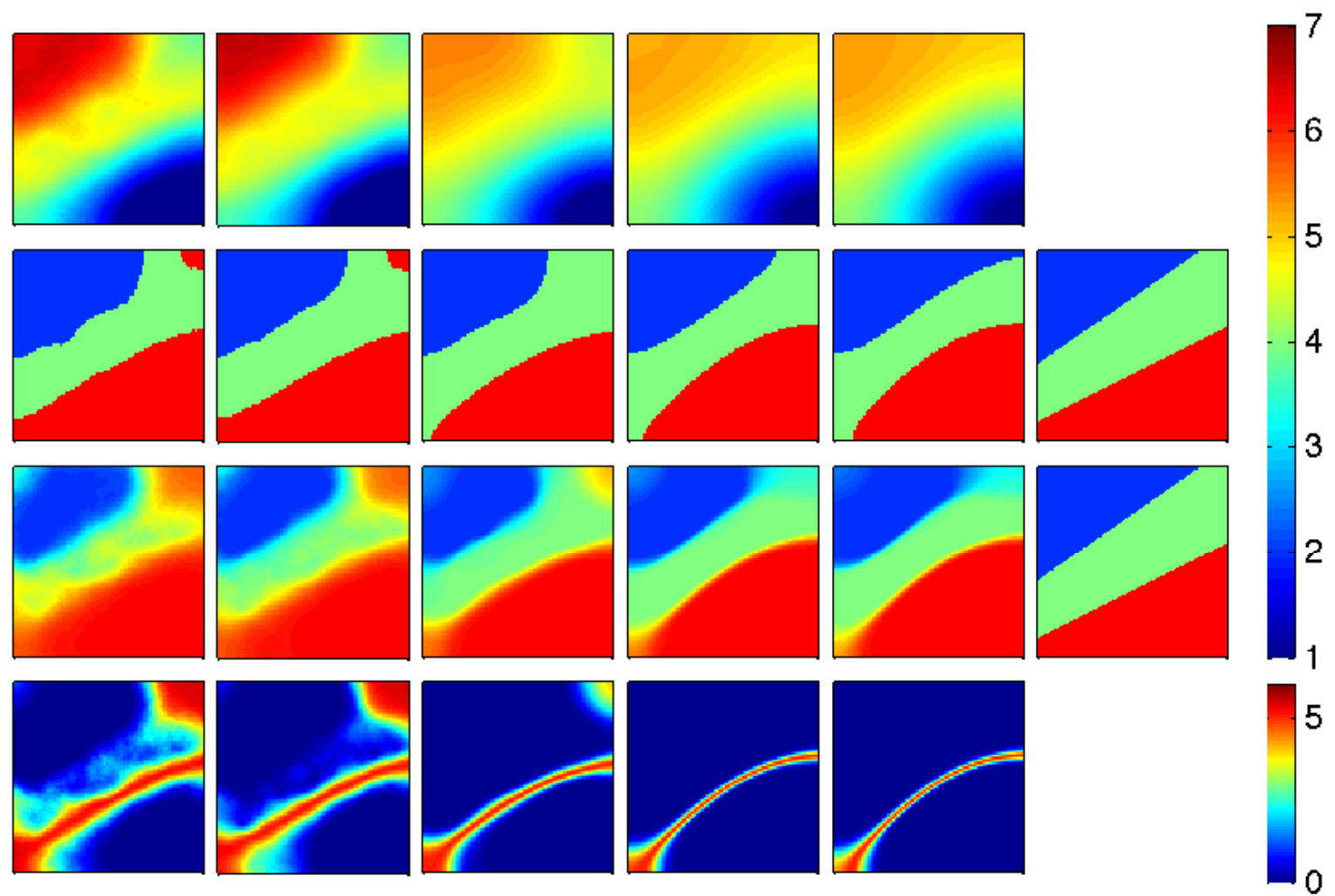

Figure 15: Identification of structural geology (layer model). MCMC results for (from left to right) $\alpha=1.5,2.0,2.5,3.0,3.5$ in the eq. (2.17). Top: MCMC mean of the level set function. Top-middle: $\kappa$ associated to the mean of the level set function (true $\kappa$ is displayed in the last column). Bottommiddle: Mean of the $\kappa$. Bottom: Variance of $\kappa$ 

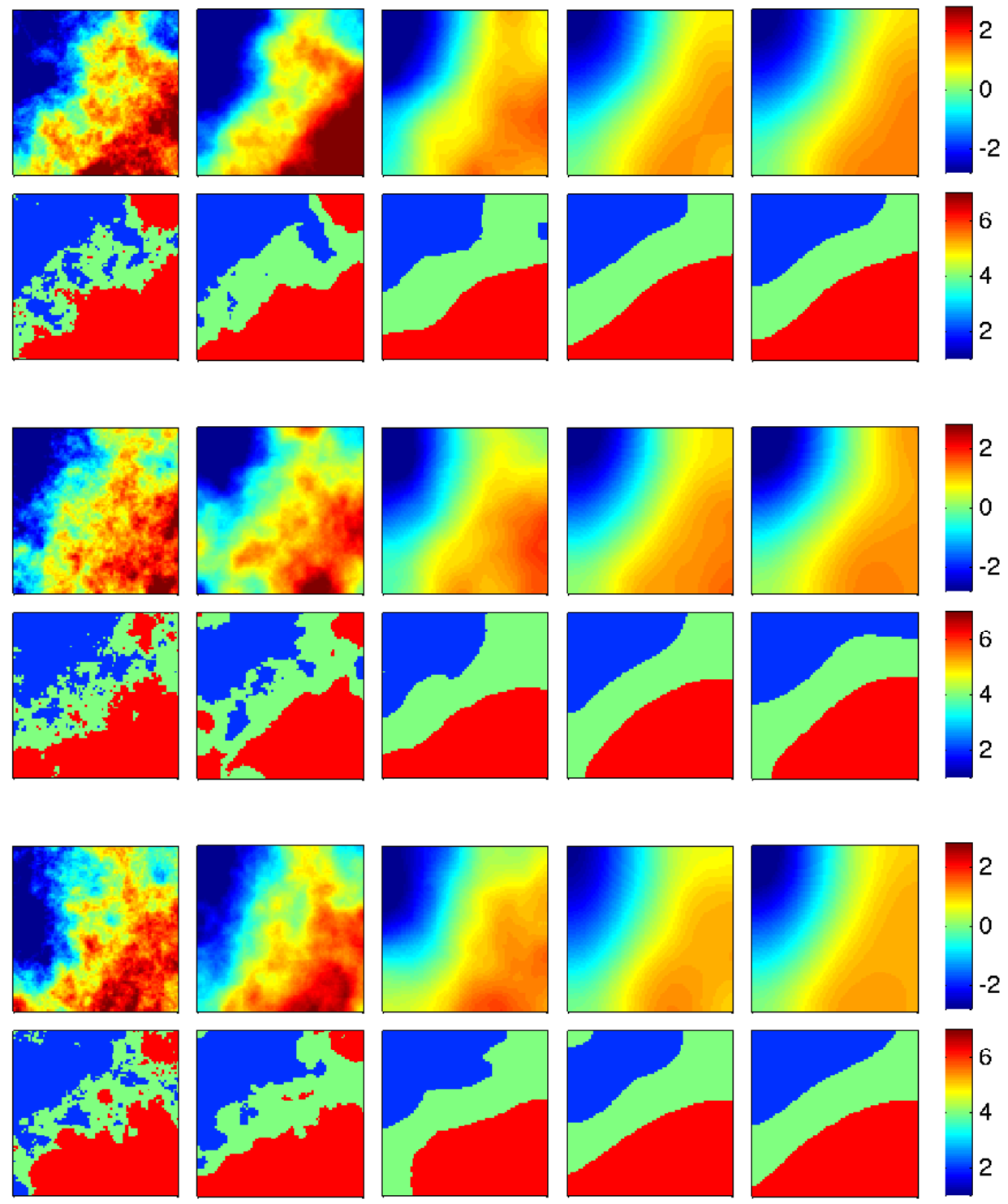

Figure 16: Identification of structural geology (layer model). Samples from the posterior on the level set (first, third and fifth rows) for (from left to right) $\alpha=1.5,2.0,2.5,3.0,3.5$ in the eq. (2.17). $\kappa$ (second, fourth and sixth rows) associated to each of these samples from the level set function. 

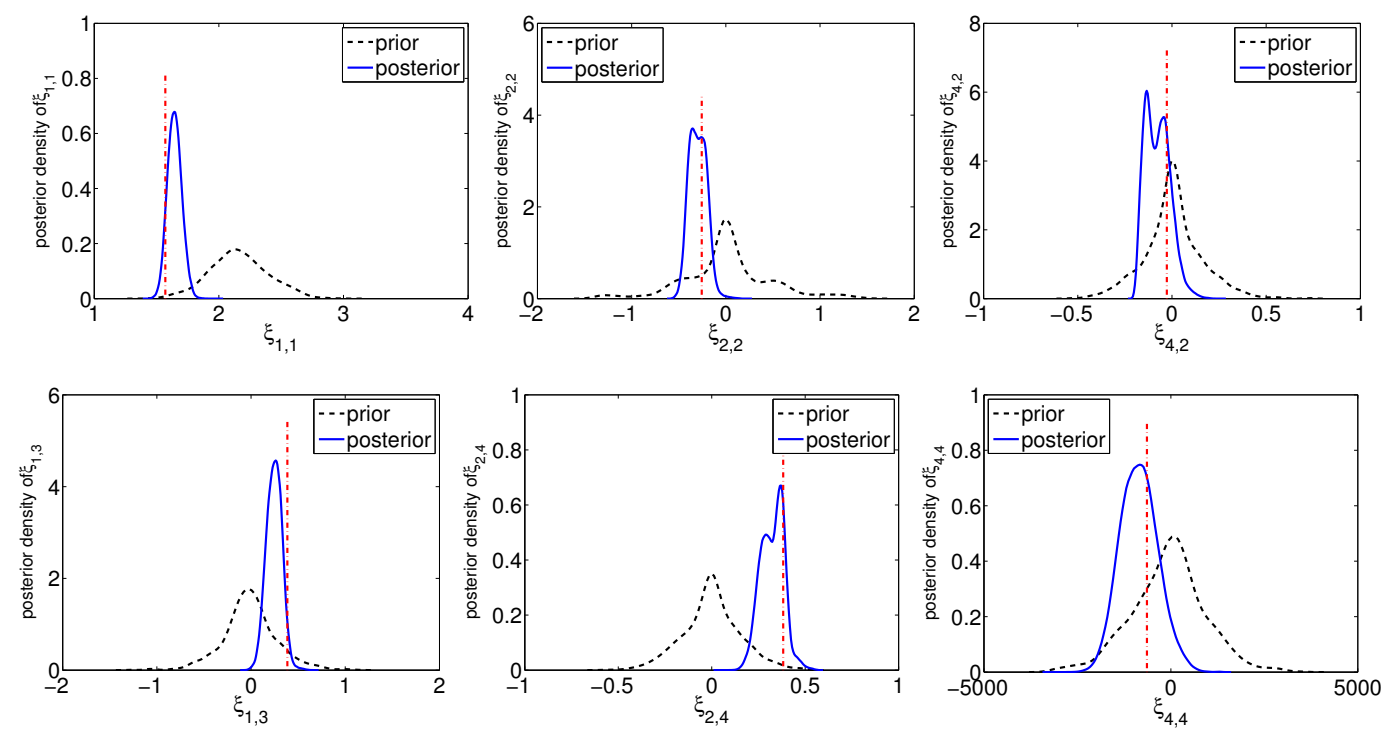

Figure 17: Identification of structural geology (layer model). Densities of the prior and posterior of some DCT coefficients of the $\kappa$ 's obtained from MCMC samples on the level set for $L=0.4$ (vertical dotted line indicates the truth).
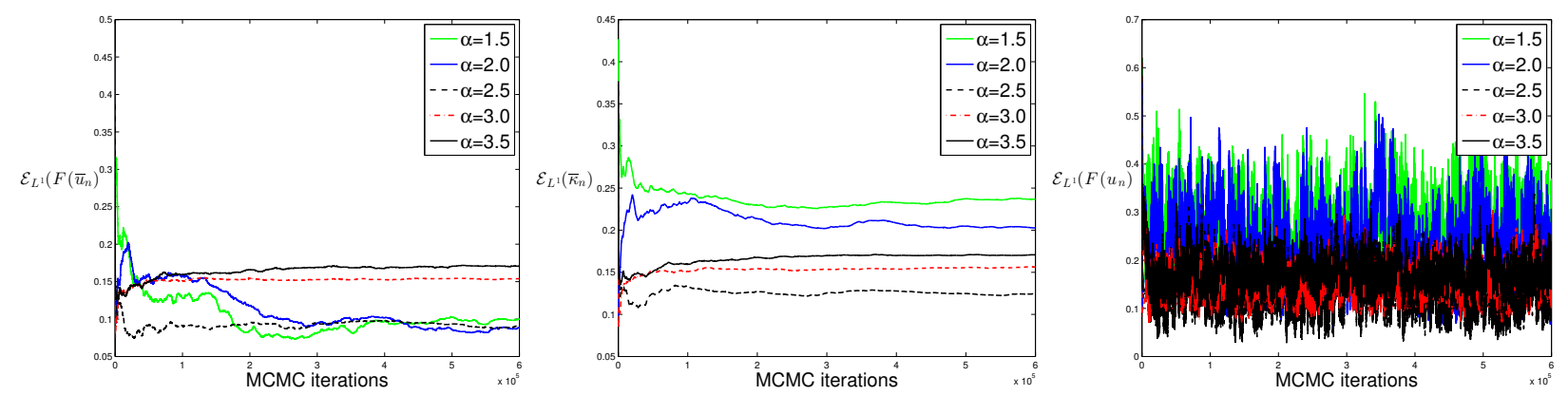

Figure 18: Identification of structural geology (layer model). $L^{1}(D)$ relative errors with respect to the truth for different choices of $\alpha$ in (2.17). Left: $\mathcal{E}_{L^{1}}\left(F\left(\bar{u}_{n}\right)\right)$. Middle: $\mathcal{E}_{L^{1}}\left(\bar{\kappa}_{n}\right)$. Right: $\mathcal{E}_{L^{1}}\left(F\left(u_{n}\right)\right)$ 
- The numerical results for the two examples that we consider demonstrate the sensitive dependence of the posterior distribution on the length-scale parameter of our Gaussian priors. It would be natural to study automatic selection techniques for this parameter, including hierarchical Bayesian modelling.

- We have assumed that the values of $\kappa_{i}$ on each unknown domain $D_{i}$ are both known and constant. It would be interesting, and possible, to relax either or both of these assumptions, as was done in the finite geometric parameterizations considered in [33].

- The numerical results also indicate that initialization of the MCMC method for the level set function can have significant impact on the performance of the inversion technique; it would be interesting to study this issue more systematically.

- The level set formulation we use here, with a single level set function and possibly multiple level set values $c_{i}$ has been used for modeling island dynamics [43] where a nested structure is assumed for the regions $\left\{D_{i}\right\}_{i=1}^{n}$ see Figure 19(a). However, we comment that there exist objects with non-nested regions, such as those depicted in Figure 19(b)-19(c), which can not be represented by a single level set function. It would be of interest to extend this work to the consideration of vector-valued level set functions. In the case of binary obstacles, it is enough to represent the shape via a single level set function (cf. [47]). However, in the case of $n$-ary obstacles or even more complex geometric objects, the representation is more complicated; see the review papers $[23,24,50]$ for more details.

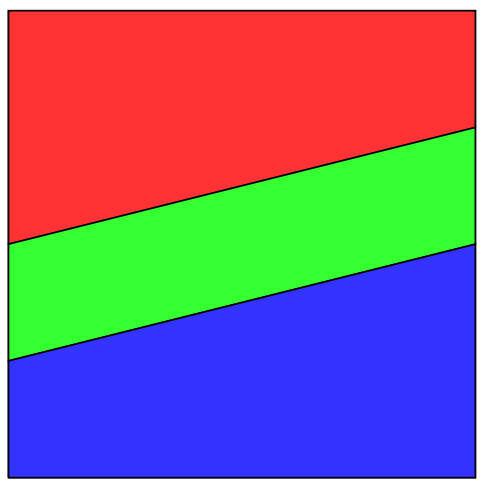

(a) Nested regions

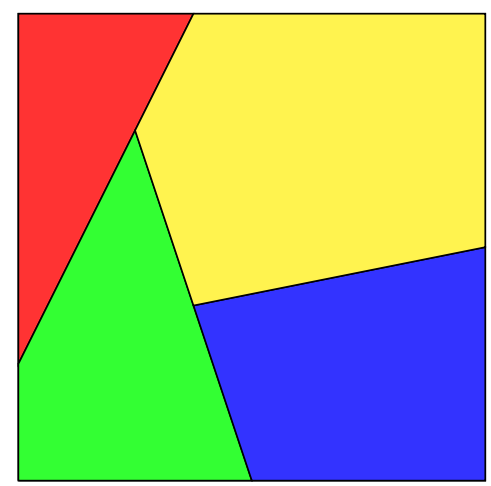

(b) Non-nested regions-I

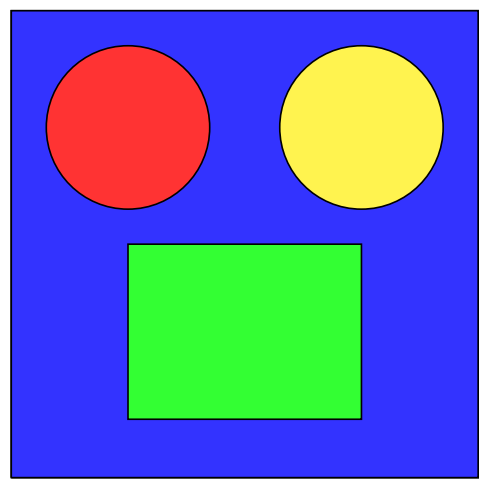

(c) Non-nested regions-II

Figure 19: Nested regions and non-nested regions

- The Bayesian framework could be potentially combined with other parameterizations of unknown geometries. For example, the pluri Gaussian approach has been used with EnKF in [40] to identify geologic facies. 


\section{Acknowledgments}

YL is is supported by EPSRC as part of the MASDOC DTC at the University of Warwick with grant No. EP/HO23364/1. AMS is supported by the (UK) EPSRC Programme Grant EQUIP, and by the (US) Office of Naval Research.

\section{References}

[1] R. J. Adler and J. E. Taylor. Random Fields and Geometry, volume 115 of Springer Monographs in Mathematics. Springer, 2007.

[2] G. Alessandrini. On the identification of the leading coefficient of an elliptic equation. Boll. Un. Mat. Ital. C (6), 4(1):87-111, 1985.

[3] H. B. Ameur, M. Burger, and B. Hackl. Level set methods for geometric inverse problems in linear elasticity. Inverse Problems, 20(3):673, 2004.

[4] S. Angenent, T. Ilmanen, and D. L. Chopp. A computed example of nonuniqueness of mean curvature flow in $\mathbf{R}^{3}$. Comm. Partial Differential Equations, 20(11-12):1937-1958, 1995.

[5] T. Arbogast, M. F. Wheeler, and I. Yotov. Mixed finite elements for elliptic problems with tensor coefficients as cell-centered finite differences. SIAM J. Numer. Anal., 34:828-852, 1997.

[6] M. Armstrong, A. Galli, H. Beucher, G. Le Loc'h, D. Renard, B. Doligez, R. Eschard, and F. Geffroy. Plurigaussian Simulations in Geosciences. 2nd revised edition edition, 2011.

[7] A. Astrakova and D.S. Oliver. Conditioning truncated pluri-Gaussian models to facies observations in ensemble-Kalman-based data assimilation. Mathematical Geosciences, page Published online April 2014, 2014.

[8] V. I. Bogachev. Gaussian Measures, volume 62 of Mathematical Surveys and Monographs. American Mathematical Society, 1998.

[9] A. Bonito, R. A. DeVore, and R. H. Nochetto. Adaptive finite element methods for elliptic problems with discontinuous coefficients. SIAM Journal on Numerical Analysis, 51(6):3106$3134,2013$.

[10] L. Borcea. Electrical impedance tomography. Inverse Problems, 18(6):R99, 2002.

[11] S.P. Brooks and A. Gelman. General methods for monitoring convergence of iterative simulations. Journal of Computational and Graphical Statistics, 7:434-455, December 1998. 
[12] Steve Brooks, Andrew Gelman, Galin Jones, and Xiao-Li Meng. Handbook of Markov Chain Monte Carlo. CRC press, 2011.

[13] T. Bui-Thanh and O. Ghattas. An analysis of infinite dimensional Bayesian inverse shape acoustic scattering and its numerical approximation. SIAM/ASA Journal on Uncertainty Quantification, 2:203-222, 2014.

[14] M. Burger. A level set method for inverse problems. Inverse Problems, 17(5):1327, 2001.

[15] M. Burger. A framework for the construction of level set methods for shape optimization and reconstruction. Interfaces and Free Boundaries, 5:301-329, 2003.

[16] M. Burger and S. Osher. A survey on level set methods for inverse problems and optimal design. Europ. J. Appl. Math., 16:263-301, 2005.

[17] J. Carrera and S. P. Neuman. Estimation of aquifer parameters under transient and steady state conditions: 3. application to synthetic and field data. Water Resources Research, 22:228-242, 1986.

[18] E. T. Chung, T. F. Chan, and X. C. Tai. Electrical impedance tomography using level set representation and total variational regularization. J. Comp. Phys., 205:357-372, 2005.

[19] S. L. Cotter, G. O. Roberts, A. M. Stuart, and D. White. MCMC methods for functions: modifying old algorithms to make them faster. Statistical Science, 28(3):424-446, 2013.

[20] S.L. Cotter, M. Dashti, and A.M. Stuart. Approximation of Bayesian inverse problems. SIAM Journal of Numerical Analysis, 48:322-345, 2010.

[21] M. Dashti and A. M. Stuart. The Bayesian approach to inverse problems. In Handbook of Uncertainty Quantification, page arxiv.1302.6989. Springer, 2016.

[22] K. Deckelnick, C. M. Elliott, and V. Styles. Double obstacle phase field approach to an inverse problem for a discontinuous diffusion coefficient. arxiv:1504.01935, 2015.

[23] O. Dorn and D. Lesselier. Level set methods for inverse scattering. Inverse Problems, 22(4):R67, 2006.

[24] O. Dorn and D. Lesselier. Level set methods for inverse scattering-some recent developments. Inverse Problems, 25(12):125001, 2009.

[25] H. W. Engl, M. Hanke, and A. Neubauer. Regularization of Inverse Problems, volume 375. Springer, 1996.

[26] L.C. Evans and J. Spruck. Motion of level sets by mean curvature I. J. Diff. Geom, 33:635-681, 1991. 
[27] F. Frühauf, O. Scherzer, and A. Leito. Analysis of regularization methods for the solution of ill-posed problems involving discontinuous operators. SIAM journal on numerical analysis, 43:767-786, 2005.

[28] M. Hanke. A regularizing Levenberg-Marquardt scheme, with applications to inverse groundwater filtration problems. Inverse Problems, 13:79-95, 1997.

[29] F. Hettlich and W. Rundell. Iterative methods for the reconstruction of an inverse potential problem. Inverse Problems, 12(3):251, 1996.

[30] M. A. Iglesias and C. Dawson. An iterative representer-based scheme for data inversion in reservoir modeling. Inverse Problems, 25:035006, 2009.

[31] M. A. Iglesias, K. Law, and A. M. Stuart. Ensemble Kalman methods for inverse problems. Inverse Problems, 29(4):045001, 2013.

[32] M. A. Iglesias, K. J. H. Law, and A. M. Stuart. Evaluation of Gaussian approximations for data assimilation in reservoir models. Computational Geosciences, 17:851-885, 2013.

[33] M. A. Iglesias, K. Lin, and A. M. Stuart. Well-posed Bayesian geometric inverse problems arising in subsurface flow. Inverse Problems, 30:114001, 2014.

[34] M. A. Iglesias and D. McLaughlin. Level-set techniques for facies identification in reservoir modeling. Inverse Problems, 27(3):035008, 2011.

[35] V. Isakov. Inverse Source Problems, volume 34 of Mathematical Surveys and Monographs. American Mathematical Society, 1990.

[36] V. Isakov. Inverse Problems for Partial Differential Equations. Applied Mathematical Sciences. Springer, 2rd edition, 2006.

[37] J. P. Kaipio and E. Somersalo. Statistical and Computational Inverse Problems, volume 160. Springer, 2005.

[38] J. Lie, M. Lysaker, and X. C. Tai. A binary level set method and some applications for mumford-shah image segmentation. IEEE Trans. Image Process., 15:1171-1181, 2006.

[39] J. Lie, M. Lysaker, and X. C. Tai. A variant of the level set method and applications to image segmentation. Math. Comput., 75:1155-1174, 2006.

[40] Ning Liu and Dean S Oliver. Ensemble Kalman filter for automatic history matching of geologic facies. Journal of Petroleum Science and Engineering, 47(3):147-161, 2005. 
[41] Nvdal G. \& Shafieirad A. Lorentzen, R. J. Estimating facies fields by use of the ensemble Kalman filter and distance functions-applied to shallow-marine environments. SPE Journal, 3:146-158., 2012.

[42] R.J. Lorentzen, K.M. Flornes, and G. Nævdal. History matching channelized reservoirs using the ensemble Kalman filter. SPE Journal, 17:137-151, 2012.

[43] B. Merriman, R. Caflisch, and S. Osher. Level set methods, with an application to modeling the growth of thin films. In Free Boundary Value Problems, Theory and Applications, pages 51-70. CRC Press, 1998.

[44] S. D. Oliver, C. A. Reynolds, and N. Liu. Inverse Theory for Petroleum Reservoir Characterization and History Matching. Cambridge University Press, 2008.

[45] J. Ping and D. Zhang. History matching of channelized reservoirs with vector-based level-set. SPE Journal, 19:514-529, 2014.

[46] G. R. Richter. An inverse problem for the steady state diffusion equation. SIAM Journal on Applied Mathematics, 41(2):210-221, 1981.

[47] F. Santosa. A level-set approach for inverse problems involving obstacles. ESAIM: Control, Optimisation and Calculus of Variations, 1:17-33, 1996.

[48] E. M. Stein and R. Shakarchi. Real Analysis : Measure Theory, Integration, and Hilbert Spaces. Princeton University Press, 2009.

[49] A. M. Stuart. Inverse problems: A Bayesian perspective. Acta Numerica, 19:451-559, 52010.

[50] X. C. Tai and T. F. Chan. A survey on multiple level set methods with applications for identifying piecewise constant functions. International Journal of Numerical Analysis and Modeling, $1: 25-47,2004$.

[51] L. A. Vese and T. F. Chan. A multiphase level set framework for image segmentation using the mumford-shah model. Int. J. Comput. Vis., 50:271-293, 2002.

[52] L. Wang, B. Sixou, and F. Peyrin. Binary tomography reconstructions with stochastic level-set methods. IEEE Signal Processing Letters, 22, 2015.

[53] J. Xie, Y. Efendiev, A. Datta-Gupta, et al. Uncertainty quantification in history matching of channelized reservoirs using markov chain level set approaches. In SPE Reservoir Simulation Symposium. Society of Petroleum Engineers, 2011. 


\section{Appendix 1}

Proof of Theorem 2.3. Notice that the random variable $y \mid u$ is distributed according to the measure $\mathbb{Q}_{u}$, which is the translate of $\mathbb{Q}_{0}$ by $\mathcal{G}(u)$, satisfying $\mathbb{Q}_{u} \ll \mathbb{Q}_{0}$ with Radon-Nikodym derivative

$$
\frac{\mathrm{d} \mathbb{Q}_{u}}{\mathrm{~d} \mathbb{Q}_{0}}(y) \propto \exp (-\Phi(u ; y))
$$

where $\Phi: U \times \mathbb{R}^{J} \rightarrow \mathbb{R}$ is the least squares function given in (2.9). Thus for the given data $y$, $\Phi(u ; y)$ is up to a constant, the negative log likelihood. We denote by $\nu_{0}$ the product measure

$$
\nu_{0}(d u, d y)=\mu_{0}(d u) \mathbb{Q}_{0}(d y) .
$$

Suppose that $\Phi(\cdot, \cdot)$ is $\nu_{0}$ measurable, then the random variable $(u, y) \in U \times Y$ is distributed according to $\nu(d u, d y)$ with

$$
\frac{\mathrm{d} \nu}{\mathrm{d} \nu_{0}}(u, y) \propto \exp (-\Phi(u ; y))
$$

From Assumptions 2.2(2) and the continuity of $\Phi(u ; y)$ with respect to $y$, we know that $\Phi(\cdot ; \cdot)$ : $U \times Y \rightarrow \mathbb{R}$ is continuous $\nu_{0}$-almost surely. Then it follows from Lemma 6.1 below that $\Phi(\cdot ; \cdot)$ is $\nu_{0}$-measurable. On the other hand, by Assumptions 2.2(1), for $|y|_{\Gamma} \leq r$, we obtain the upper and lower bound for $Z$,

$$
0<\exp \left(-K\left(r, \kappa_{\min }\right)\right) \leq Z=\int_{U} \exp (-\Phi(u ; y)) \mu_{0}(d u) \leq 1
$$

Thus the measure is normalizable and applying the Bayes' Theorem 3.4 from [21] yields the existence of $\mu^{y}$.

Let $Z=Z(y)$ and $Z^{\prime}=Z\left(y^{\prime}\right)$ be the normalization constants for $\mu^{y}$ and $\mu^{y^{\prime}}$, i.e.

$$
Z=\int_{U} \exp (-\Phi(u ; y)) \mu_{0}(d u), \quad Z^{\prime}=\int_{U} \exp \left(-\Phi\left(u ; y^{\prime}\right)\right) \mu_{0}(d u)
$$

We have seen above that

$$
1 \geq Z, Z^{\prime} \geq \exp \left(-K\left(r, \kappa_{\min }\right)\right)>0 .
$$

From Assumptions 2.2(3),

$$
\left|Z-Z^{\prime}\right| \leq \int\left|\exp (-\Phi(u ; y))-\exp \left(-\Phi\left(u ; y^{\prime}\right)\right)\right| \mu_{0}(\mathrm{~d} u) \leq \int\left|\Phi(u ; y)-\Phi\left(u ; y^{\prime}\right)\right| \mu_{0}(\mathrm{~d} u) \leq C(r)\left|y-y^{\prime}\right|_{\Gamma}
$$

Thus, by the definition of Hellinger distance, we have

$$
2 d_{\mathrm{Hell}}\left(\mu^{y}, \mu^{y^{\prime}}\right)^{2}=\int\left(Z^{-1 / 2} \exp \left(-\frac{1}{2} \Phi(u ; y)\right)-\left(Z^{\prime}\right)^{-1 / 2} \exp \left(-\frac{1}{2} \Phi\left(u ; y^{\prime}\right)\right)\right)^{2} \mu_{0}(\mathrm{~d} u) \leq I_{1}+I_{2}
$$

where

$$
\begin{aligned}
& I_{1}=\frac{2}{Z} \int\left(\exp \left(-\frac{1}{2} \Phi(u ; y)\right)-\exp \left(-\frac{1}{2} \Phi\left(u ; y^{\prime}\right)\right)\right)^{2} \mu_{0}(\mathrm{~d} u) \\
& I_{2}=2\left|Z^{-1 / 2}-\left(Z^{\prime}\right)^{-1 / 2}\right|^{2} \int \exp \left(-\Phi\left(u ; y^{\prime}\right)\right) \mu_{0}(\mathrm{~d} u)
\end{aligned}
$$


Applying (1) and (2) in Assumptions 2.2 again yields

$$
\frac{Z}{2} I_{1} \leq C(r)\left|y-y^{\prime}\right|_{\Gamma}^{2}
$$

and

$$
I_{2} \leq C(r)\left|Z^{-1 / 2}-\left(Z^{\prime}\right)^{-1 / 2}\right|^{2} \leq C(r)\left(Z^{-3} \vee\left(Z^{\prime}\right)^{-3}\right)\left|Z-Z^{\prime}\right|^{2} \leq C(r)\left|y-y^{\prime}\right|_{\Gamma}^{2}
$$

Therefore the proof that the measure is Lipschitz is completed by combining the preceding estimates. The final statement follows as in [49], after noting that $f \in L_{\mu_{0}}^{2}(U ; S)$ implies that $f \in$ $L_{\mu^{y}}^{2}(U ; S)$, since $\Phi(\cdot ; y) \geq 0$.

Lemma 6.1. Let $U$ be a separable Banach space and $(U, \Sigma, \mu)$ be a complete probability space with $\sigma$-algebra $\Sigma$. If a functional $\mathcal{F}: U \rightarrow \mathbb{R}$ is continuous $\mu$-almost surely, i.e. $\mu(M)=1$ where $M$ denotes the set of the continuity points of $\mathcal{F}$, then $\mathcal{F}$ is $\Sigma$-measurable.

Proof. By the definition of measurability, it suffices to show that for any $c>0$

$$
S:=\{u \in U \mid \mathcal{F}(u)>c\} \in \Sigma .
$$

One can write $S$ as $S=(S \cap M) \cup(S \backslash M)$. Since $\mathcal{F}$ is continuous $\mu$-almost surely, $M$ is measurable and $\mu(M)=1$. It follows from the completeness of the measure $\mu$ that $S \backslash M$ is measurable and $\mu(S \backslash M)=0$. Now we claim that $S \cap M$ is also measurable. Denote $B_{\delta}(u) \subset U$ to be the ball of radius $\delta$ centered at $u \in U$. For each $v \in S \cap M$, as $\mathcal{F}$ is continuous at $v$, there exists $\delta_{v}>0$ such that if $v^{\prime} \in B_{\delta_{v}}(v)$, then $\mathcal{F}\left(v^{\prime}\right)>c$. Therefore $S \cap M$ can be written as

$$
S \cap M=M \bigcap \bigcup_{v \in S \cap M} B_{\delta_{v}}(v)
$$

that is the intersection of the measurable set $M$ with the open set $\bigcup_{v \in S \cap M} B_{\delta_{v}}(v)$. So $S \cap M$ is measurable. Then it follows that $\mathcal{F}$ is $\Sigma$-measurable.

\section{Appendix 2}

Recall the Gaussian measure $\mu_{0}=\mathcal{N}(0, \mathcal{C})$ on the function space $\mathcal{H}$ where $\mathcal{C}=\mathcal{C}_{i}, \mathcal{H}=\mathcal{H}_{i}, i=1,2$ given in subsection 2.5. These measures can be constructed as Gaussian random fields.

Let $(\Omega, \mathscr{F}, \mathbb{P})$ be a complete probability space, i.e. if $A \in \mathscr{F}$ with $\mathbb{P}(A)=0$, then $\mathbb{P}(B)=0$ for $B \subset A$. A random field on $D$ is a measurable mapping $u: D \times \Omega \rightarrow \mathbb{R}$. Thus, for any $x \in D, u(x ; \cdot)$ is a random variable in $\mathbb{R}$; whilst for any $\omega \in \Omega, u(\cdot ; \omega): D \rightarrow \mathbb{R}$ is a vector field. Denote by $\left(\mathbb{R}^{\mathbb{N}}, \mathcal{B}\left(\mathbb{R}^{\mathbb{N}}\right), \mathbb{P}\right)$ the probability space of i.i.d standard Gaussian sequences equipped with product $\sigma$-algebra and product measure. In this paper, we consider $(\Omega, \mathscr{F}, \mathbb{P})$ as the completion of $\left(\mathbb{R}^{\mathbb{N}}, \mathcal{B}\left(\mathbb{R}^{\mathbb{N}}\right), \mathbb{P}\right)$ in which case the $\sigma$-algebra $\mathscr{F}$ consists of all sets of the type $A \cup B$, where $A \in \mathcal{B}\left(\mathbb{R}^{\mathbb{N}}\right)$ and $B \subset N \in \mathcal{B}\left(\mathbb{R}^{\mathbb{N}}\right)$ with $\mathbb{P}(N)=0$. Let $\omega=\left\{\xi_{k}\right\}_{k=1}^{\infty} \in \Omega=\mathbb{R}^{\mathbb{N}}$ be an i.i.d sequence 
with $\xi_{1} \sim \mathcal{N}(0,1)$, and consider the random function $u \in \mathcal{H}$ defined via the Karhunen-Loéve expansion

$$
u(x ; \omega)=\mathcal{T}(\omega):=\sum_{k=1}^{\infty} \sqrt{\lambda_{k}} \xi_{k} \phi_{k}(x),
$$

where $\left\{\lambda_{k}, \phi_{k}\right\}_{k=1}^{\infty}$ is the eigensystem of $\mathcal{C}$. By the theory of Karhunen-Loéve expansions [8], the law of the random function $u$ is identical to $\mu_{0}$. Recalling that $\alpha>1$, the eigenvalues $\left\{\lambda_{k}\right\}$ of $\mathcal{C}_{1}$ decay like $k^{-\alpha}$ in two dimensions; whilst the eigenvalues of $\mathcal{C}_{2}$ will decay exponentially. Moreover, we assume further that $\phi_{k} \in U$ and that $\sup _{k}\left\|\phi_{k}\right\|_{\infty}<\infty$ which holds in simple geometries. Due to the decaying properties of the eigenvalues of $\mathcal{C}$, the truncated sum

$$
\mathcal{T}_{N}(\omega)=\sum_{k=1}^{N} \sqrt{\lambda_{k}} \xi_{k} \phi_{k}
$$

admits a limit $\mathcal{T}$ in $L_{\mathbb{P}}^{2}(\Omega ; \mathcal{H})$. By the Kolmogorov Continuity Theorem [21], $\mathcal{T}$ is in fact Hölder continuous $\mathbb{P}$-almost surely; in particular, $\mathcal{T} \in U \mathbb{P}$-almost surely. Then by Theorem 3.1.2 in [1], we have $\mathcal{T}_{N} \rightarrow \mathcal{T}$ in the uniform norm of $U, \mathbb{P}$-almost surely. Since for any $N \in, \mathcal{T}_{N}:(\Omega, \mathscr{F}) \rightarrow$ $(U, \mathscr{B}(U))$ is continuous and thus measurable, we know from the completeness of $(\Omega, \mathscr{F}, \mathbb{P})$ that the limit $\mathcal{T}$ is also measurable from $(\Omega, \mathscr{F})$ to $(U, \mathscr{B}(U))$ (see p30 in [48]). The measurability of $\mathcal{T}$ enables us to define a new measure on $(U, \mathscr{B}(U))$ which we still denote by $\mu_{0}$ by the following:

$$
\mu_{0}(A)=\mathbb{P}\left(\mathcal{T}^{-1}(A)\right)=\mathbb{P}(\{\omega \in \Omega \mid u(\cdot ; \omega) \in A\}) \quad \text { for } A \in \mathscr{B}(U) .
$$

Thus $\mu_{0}$ is indeed the push-forward measure of $\mathbb{P}$ through $\mathcal{T}$. By definition, it is not hard to verify that $\mu_{0}$ is the Gaussian measure $\mathcal{N}(0, \mathcal{C})$ on $(U, \mathscr{B}(U))$. In addition, suppose that $B \subset N \in \mathscr{B}(U)$ with $\mu_{0}(N)=0$; if we still define $\mu_{0}(B)$ according to (7.3), then $\mu_{0}(B)=\mathbb{P}\left(\mathcal{T}^{-1}(B)\right)=0$ by the fact that $\mathcal{T}^{-1}(B) \subset \mathcal{T}^{-1}(N)$ and the completeness of $(\Omega, \mathscr{F}, \mathbb{P})$. Denote by $\Sigma$ the smallest $\sigma$ algebra containing $\mathscr{B}(U)$ and all sets of zero measure under $\mu_{0}$ so that any set $E \in \Sigma$ is of the form $E=A \cup B$, where $A \in \mathscr{B}(U)$ and $B \subset N \in \mathscr{B}(U)$ with $\mu_{0}(N)=0$. Then $\left(U, \Sigma, \mu_{0}\right)$ is complete.

We comment that although a Gaussian measure is usually defined as a Borel measure in the literature (see e.g. [8]), it is more convenient to work with a complete Gaussian measure in this paper; in particular, the completeness of $\mu_{0}$ is employed to show the measurability of the observational map in level set based inverse problems.

Considering a Gaussian random function $u(\cdot ; \omega)$ with $\omega \in \Omega$, for any level constant $c \in \mathbb{R}$, we define the random level set

$$
D_{c}^{0}=D_{c}^{0}(u(\cdot ; \omega))=D_{c}^{0}(\omega):=\{x \mid u(x ; \omega)=c\} .
$$

Recall that the measure space $\left(U, \Sigma, \mu_{0}\right)$ is the push-forward of $(\Omega, \mathscr{F}, \mathbb{P})$ under $\mathcal{T}$. We define the functional $\mathcal{M}_{c}: U \rightarrow \mathbb{R}$ by

$$
\mathcal{M}_{c} u=m(\{x \mid u(x)=c\})
$$


and the composition $\mathcal{R}_{c}:=\mathcal{M}_{c} \circ \mathcal{T}$, as illustrated in the following commutative diagram:

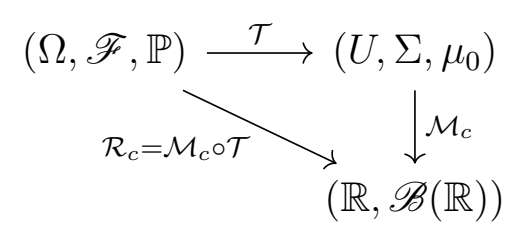

Lemma 7.1. For any $c \in \mathbb{R}, \mathcal{M}_{c}$ is $\Sigma$-measurable and $\mathcal{R}_{c}$ is $\mathscr{F}$-measurable so that $m\left(D_{c}^{0}\right)$ is a random variable on both $\left(U, \Sigma, \mu_{0}\right)$ and $(\Omega, \mathscr{F}, \mathbb{P})$.

Proof. To prove $\mathcal{M}_{c}$ is $\Sigma$-measurable, we only need to check the set $A_{t}:=\left\{u \in U \mid \mathcal{M}_{c} u \geq t\right\} \in \Sigma$ for any $t \in \mathbb{R}$. Since $\mathcal{M}_{c}$ is a non-negative map, for $t \leq 0$, it is obvious that $A_{t}=U$ and hence measurable. Now we claim that $A_{t}$ is closed in $U$ for $t>0$. To that end, let $\left\{u_{n}\right\}_{n=1}^{\infty}$ be a sequence of functions in $A_{t}$ such that $\left\|u_{n}-u\right\|_{U} \rightarrow 0$ for some $u \in U$ as $n \rightarrow \infty$. We prove that $u \in A_{t}$. Since $\left\|u_{n}-u\right\|_{U} \rightarrow 0$, there exists a subsequence which is still denoted by $u_{n}$ such that $\left\|u_{n}-u\right\|_{U}<1 / n$. By the definition of $A_{t}, u_{n} \in A_{t}$ means that $m\left(\left\{x \in \bar{D} \mid u_{n}(x)=c\right\}\right) \geq t$ for all $n$. Moreover, from the construction of $u_{n},\left\{x \in \bar{D} \mid u_{n}(x)=c\right\} \subset B_{n}:=\{x \in \bar{D}|| u(x)-c \mid<1 / n\}$, which implies that $m\left(B_{n}\right) \geq t$. Noting that

$$
\{x \in \bar{D} \mid u(x)=c\}=\cap_{n=1}^{\infty} B_{n}
$$

and that $B_{n}$ is decreasing, we can conclude that $m(\{x \in \bar{D} \mid u(x)=0\}) \geq t$, i.e. $u \in A_{t}$. So $A_{t}$ is closed for $t>0$. Then it follows from the measurability of $\mathcal{T}$ that $\mathcal{R}_{c}$ is $\mathscr{F}$-measurable. Therefore $m\left(D_{c}^{0}\right)$ is a random variable on both $\left(U, \Sigma, \mu_{0}\right)$ and $(\Omega, \mathscr{F}, \mathbb{P})$.

The following theorem demonstrates that $m\left(D_{c}^{0}\right)$ vanishes almost surely on both measure spaces above.

Proposition 7.2. Consider a random function $u$ drawn from one of the Gaussian probability measures $\mu_{0}$ on $(U, \Sigma)$ given in subsection 2.5. For $c \in \mathbb{R}$, the random level set $D_{c}^{0}$ of $u$ is defined by (7.4). Then

(i) $m\left(D_{c}^{0}\right)=0, \mathbb{P}$-almost surely;

(ii) $m\left(D_{c}^{0}\right)=0, \mu_{0}$-almost surely.

Proof. (i) For any fixed $x \in D$, since the point evaluation $u(x)$ acts as a bounded linear functional on $U, u(x ; \cdot)$ is a real valued Gaussian random variable, which implies $\mathbb{P}(\{\omega \mid u(x, \omega)=c\})=0$. Moreover, noting that the random field $u: D \times \Omega \rightarrow \mathbb{R}$ is a measurable map, if we view $m\left(D_{c}^{0}\right)$ as a random variable on $\Omega$, then

$$
\begin{array}{r}
\mathbb{E}\left[m\left(D_{c}^{0}\right)\right]=\int_{\Omega} m\left(D_{c}^{0}(\omega)\right) \mathrm{d} \mathbb{P}(\omega)=\int_{\Omega} \int_{\mathbb{R}^{d}} \mathbb{I}_{\{x \mid u(x ; \omega)=c\}} \mathrm{d} x \mathrm{~d} \mathbb{P}(\omega) \\
=\int_{\Omega} \int_{\mathbb{R}^{d}} \mathbb{I}_{\{(x, \omega) \mid u(x ; \omega)=c\}} \mathrm{d} x \mathrm{~d} \mathbb{P}(\omega) \stackrel{\text { Fubini }}{=} \int_{\mathbb{R}^{d}} \int_{\Omega} \mathbb{I}_{\{(x, \omega) \mid u(x ; \omega)=c\}} \mathrm{d} \mathbb{P}(\omega) \mathrm{d} x \\
=\int_{\mathbb{R}^{d}} \int_{\Omega} \mathbb{I}_{\{\omega \mid u(x ; \omega)=c\}} \mathrm{d} \mathbb{P}(\omega) \mathrm{d} x=\int_{\mathbb{R}^{d}} \mathbb{P}(\{\omega \mid u(x ; \omega)=c\}) \mathrm{d} x=0
\end{array}
$$


Noting that $m\left(D_{c}^{0}\right) \geq 0$, we obtain $m\left(D_{c}^{0}\right)=0, \mathbb{P}$-almost surely.

(ii) Recall that $A_{t}=\left\{u \in U \mid \mathcal{M}_{c} u \geq t\right\}$ defined in Lemma 7.1 is closed in $U$ for any $t>0$. Thus the set $A:=\{u \in U \mid m(\{x \mid u(x)=c\})=0\}=\left(\cup_{k=1}^{\infty} A_{1 / k}\right)^{c}=\cap_{k=1}^{\infty} A_{1 / k}^{c}$ is a Borel set of $U$ and measurable. Since $\mu_{0}$ is the push-forward measure of $\mathbb{P}$ under $\mathcal{T}$,

$$
\mu_{0}(A)=\mathbb{P}\left(\mathcal{T}^{-1}(A)\right)=\mathbb{P}\left(\left\{\omega \mid m\left(D_{c}^{0}(\omega)\right)=0\right\}\right)=1
$$

where the last equality follows from (i). 